\title{
Sismicidad de interior de placa en Cuba
}

\author{
Mario Octavio Cotilla Rodríguez*
}

Recibido en octubre de 2013; aceptado en marzo de 2014

\begin{abstract}
The greatest territorial area of Cuba is related with the intraplate seismicity of the North American plate. This territory is defined in the West Neotectonic Unit with $\sim 900$ perceptible earthquakes (1492-2013). The maximum magnitude value estimated is 6.2 , earthquakes of 1880 and 1914. The strongest earthquakes show a recurrence period upper 100 years. These events have some casualties and injured, but much less than of the Southeastern Cuban region ( 100). Seismic activity is justified by the dynamic influence of the Caribbean-North American plate boundary on the fault zones and their intersections.
\end{abstract}

Key words: Active faults, Caribbean, Cuba, earthquakes, seismicity.

\section{Resumen}

La mayor extensión superficial de Cuba está relacionada con la sismicidad interior de la placa de Norteamérica. Este territorio se define en la Unidad Neotectónica Occidental con $\sim 900$ terremotos perceptibles (1492-2013). El valor de la magnitud máxima estimada es 6.2, años 1880 y 1914. Los terremotos más fuertes demuestran un periodo de recurrencia superior a 100 años. El conjunto de todos los eventos ha producido algunos muertos y heridos, pero mucho menos que los de la región suroriental ( 100). La actividad sísmica se justifica por la influencia dinámica del límite de placas Caribe-Norteamérica en las zonas de fallas y sus intersecciones.

Palabras clave: Caribe, Cuba, fallas activas, sismicidad, terremotos.

\section{Introducción}

Cuba es un archipiélago $\left(\sim 111,000 \mathrm{~km}^{2}\right)$ en el norte del Mar Caribe (Figura 1), desde el punto de vista físico-geográfico, está integrada en el Arco de Islas de las Antillas Mayores. El área de Cuba es mayor que la correspondiente del conjunto de las islas caribeñas del mencionado Arco $\left(\sim 96,000 \mathrm{~km}^{2}\right)$, es también la más larga de las islas $(\sim 1,260 \mathrm{~km})$ y su longitud de costa es de $\sim 5,750 \mathrm{~km}$. La plataforma insular que la rodea alcanza $\sim 68,000 \mathrm{~km}^{2}$ y la profundidad está en $10-55 \mathrm{~m}$, el talud insular es de $\sim 5 \mathrm{~km}$ de profundidad, en la parte suroriental se encuentra una fosa oceánica

* Departamento de Física de la Tierra, Astronomía y Astrofísica I, Facultad de Ciencias Físicas, Universidad Complutense de Madrid, Ciudad Universitaria, s/n, 28040, Madrid, correo electrónico: macot@fisi.ucm.es; dcordoba@fisi.ucm.es 


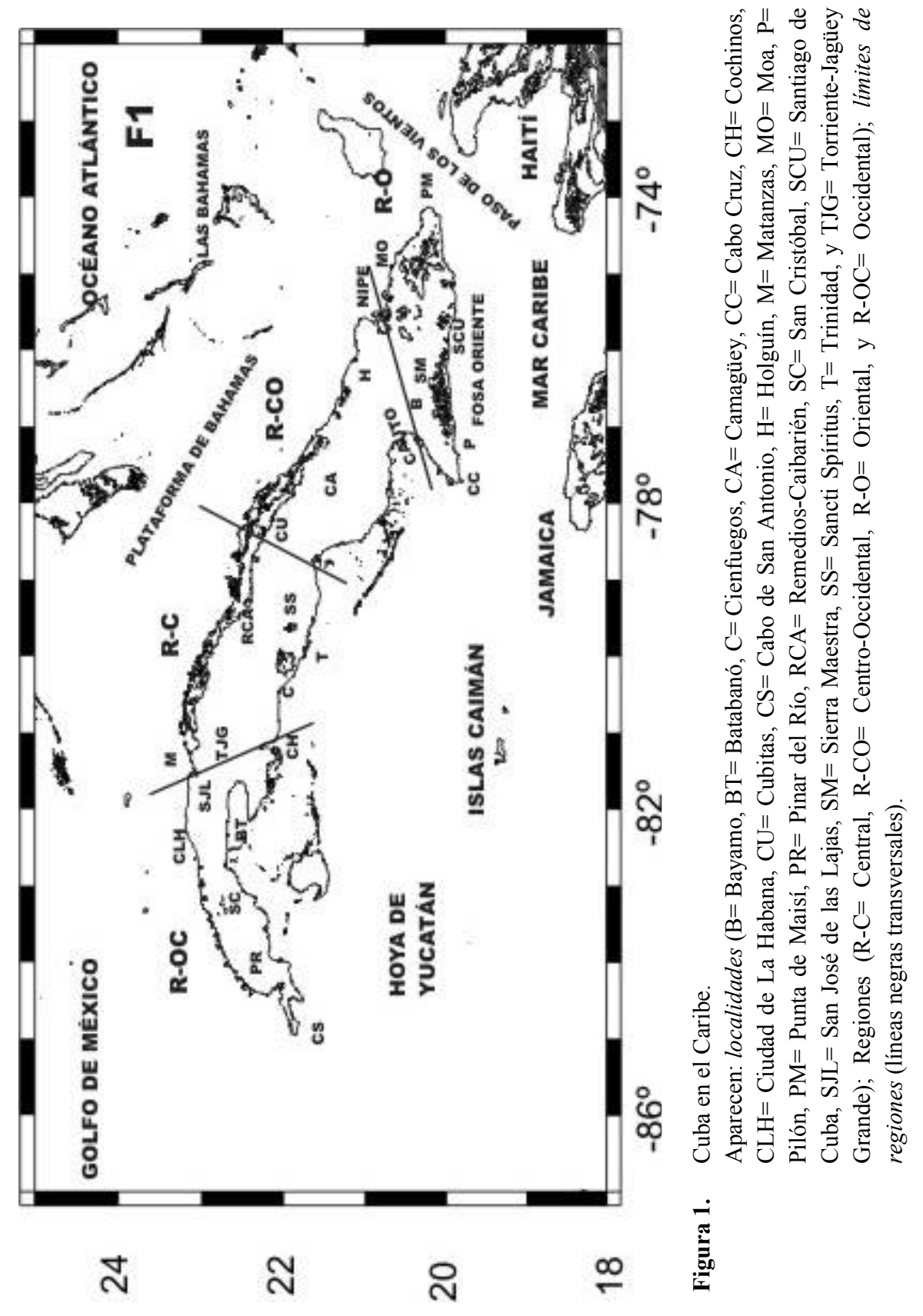


(Oriente, de $\sim 7,680 \mathrm{~m}$ ). En esta zona se produce un contraste de $\sim 10 \mathrm{~km}$ de desnivel entre la Sierra Maestra (Pico Turquino, $\mathrm{h}=1,974 \mathrm{~m}$ ) y la fosa adyacente, en una distancia de apenas $5 \mathrm{~km}$.

La actividad sísmica (AS) del Caribe se ha reconocido desde la llegada de los españoles (Figura 2a). En particular destacan, por la energía y la cantidad de terremotos, los segmentos de Haití, Jamaica, República Dominicana, Puerto Rico y la parte suroriental de Cuba (Figura 2a). Para el caso de Cuba, la colonización española comenzó de oriente a occidente (Tabla 1). Y la AS fue, consecuentemente, conocida en la zona Oriental (ciudades de Baracoa, Santiago de Cuba y Bayamo, principalmente) (Figura 1). Sin embargo, hay al menos una leyenda de los pobladores nativos de entonces sobre los terremotos, pero de la parte occidental, específicamente de Cuba Occidental (CO). En ella se explica la configuración de la desembocadura del río Yumurí, una zona aledaña a la bahía de Matanzas (Figura 3), a partir de la relación amorosa de dos jóvenes nativos. En el catálogo de Poey aparece que en 1842 esta zona tuvo un maremoto. Ese evento se corresponde, muy probablemente, con el fuerte terremoto de Cabo Haitiano del 7 de mayo de 1842 (Figura 2a). Matanzas está lejos de la región suroriental; sin embargo, ha tenido reportes de algunos de los fuertes sismos que se produjeron en Santiago de Cuba. Específicamente, Matanzas se localiza en la Unidad Neotectónica Occidental (UNOC) (Figura 3) y la zona fue habitada desde 1693.

La sismicidad en Cuba tiene características muy distintas en, al menos, dos zonas: 1) suroriental (coincidente con el límite entre las placas Norteamérica y Caribe); 2) resto del territorio (incluido en la placa de Norteamérica) (Figura 2b). Es evidente que la primera de ellas es más activa y conlleva un mayor peligro. Esto fue reconocido por el Padre Benito Viñes Martorell, S.J. (Gutiérrez Lanza, 1910, 1914). También Morales y Pedroso (1931) aseguró que los terremotos de: 1) CO son de origen volcánico y tienen su hipocentro en América Central; 2) la parte Oriental de Cuba son de origen tectónico. No obstante, la mayor parte de la población, incluso en la actualidad, considera que es en Cuba Oriental, y en específico Santiago de Cuba, donde único se producen los terremotos. Así que nuestro objetivo se dirige a la sismicidad de $\mathrm{CO}$.

Para el caso de $\mathrm{CO}$ hay documentados muchos terremotos, pero muy espaciados en tiempo, y con magnitudes relativamente bajas, en $\mathrm{CO}$ han ocurrido dos terremotos fuertes $(\mathrm{M} \geq 6)$ en Pinar del Río y Holguín (Figura 4). La mayoría de los terremotos de la UNOC no son perceptibles, las determinaciones instrumentales son muy escasas, aunque mejoran con el incremento de estaciones sísmicas permanentes, en este sentido hay un importante contraste con las informaciones sobre los eventos sísmicos de la parte suroriental. Se define entonces la sismicidad de la primera región como de interior de placa (SIP). 

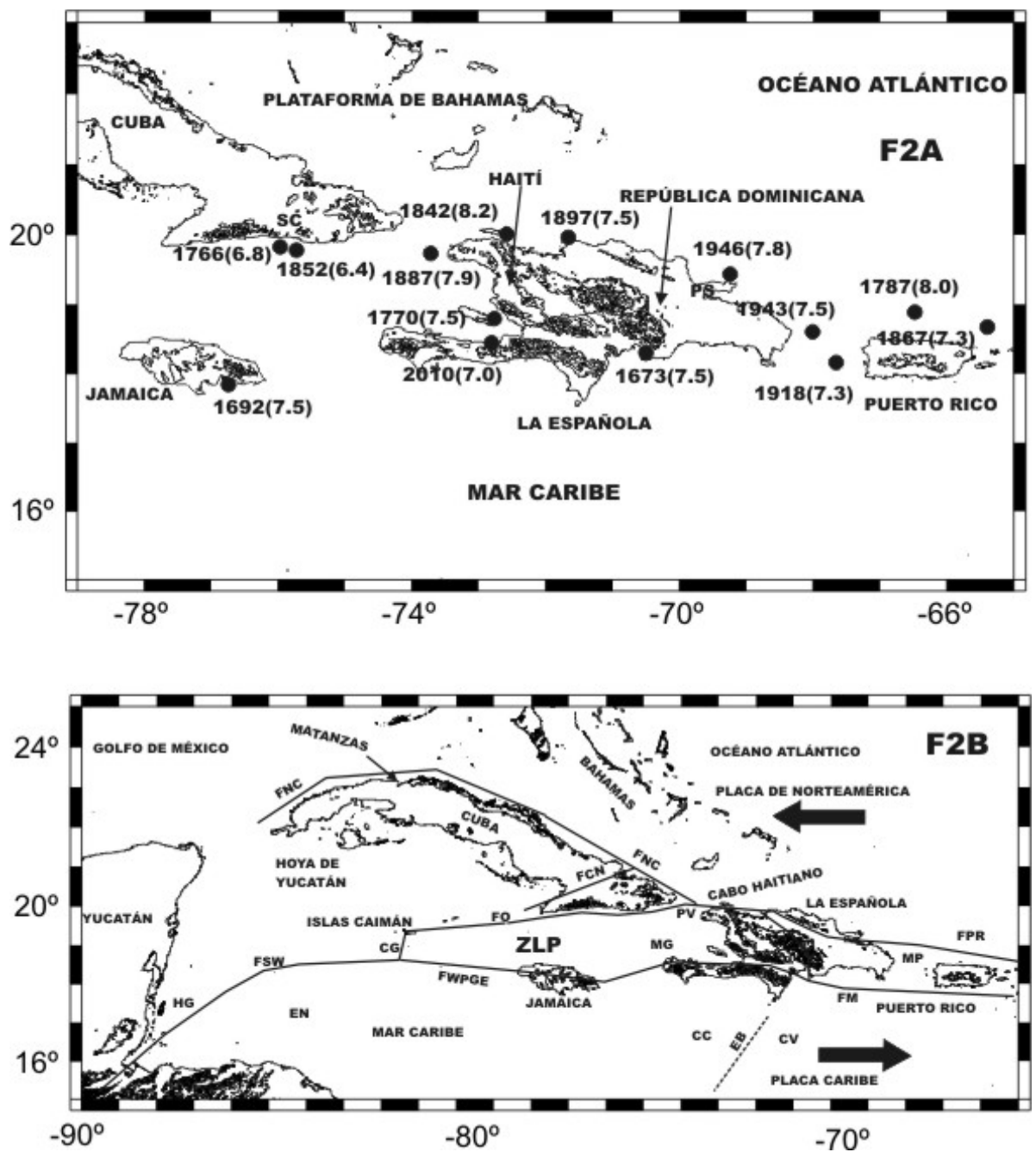

Figura 2a. Terremotos fuertes del Caribe Norte, inmediatos a Cuba.

Aparecen: epicentros (círculos negros); año de ocurrencia: 1852; magnitud: 7.5, localidades: $\mathrm{SC}=$ Santiago de Cuba, PS= Península de Samaná.

Figura 2b. Tectónica del Caribe Norte.

Aparecen: flechas negras gruesas (sentido de movimiento de las placas) $\mathrm{ZLP}=$ Zona límite de placas; líneas negras continuas (fallas principales: $\mathrm{FCN}=$ CautoNipe, $\mathrm{FNC}=$ Nortecubana, $\mathrm{FO}=$ Oriente, $\mathrm{FSW}=$ Swan-Walton, $\mathrm{FWPGE}=$ Walton-Gonave); localidades y lugares ( $\mathrm{CC}=$ Cuenca de Colombia, $\mathrm{CG}=$ Centro de Generación, $\mathrm{CV}=$ Cuenca de Venezuela, $\mathrm{EB}=$ Elevado de Beata, EN= Elevado de Nicaragua, FM= Fosa de Muertos, FPR= Fosa de Puerto Rico, HG= Hoya de Guatemala, y PV= Paso de los Vientos). 


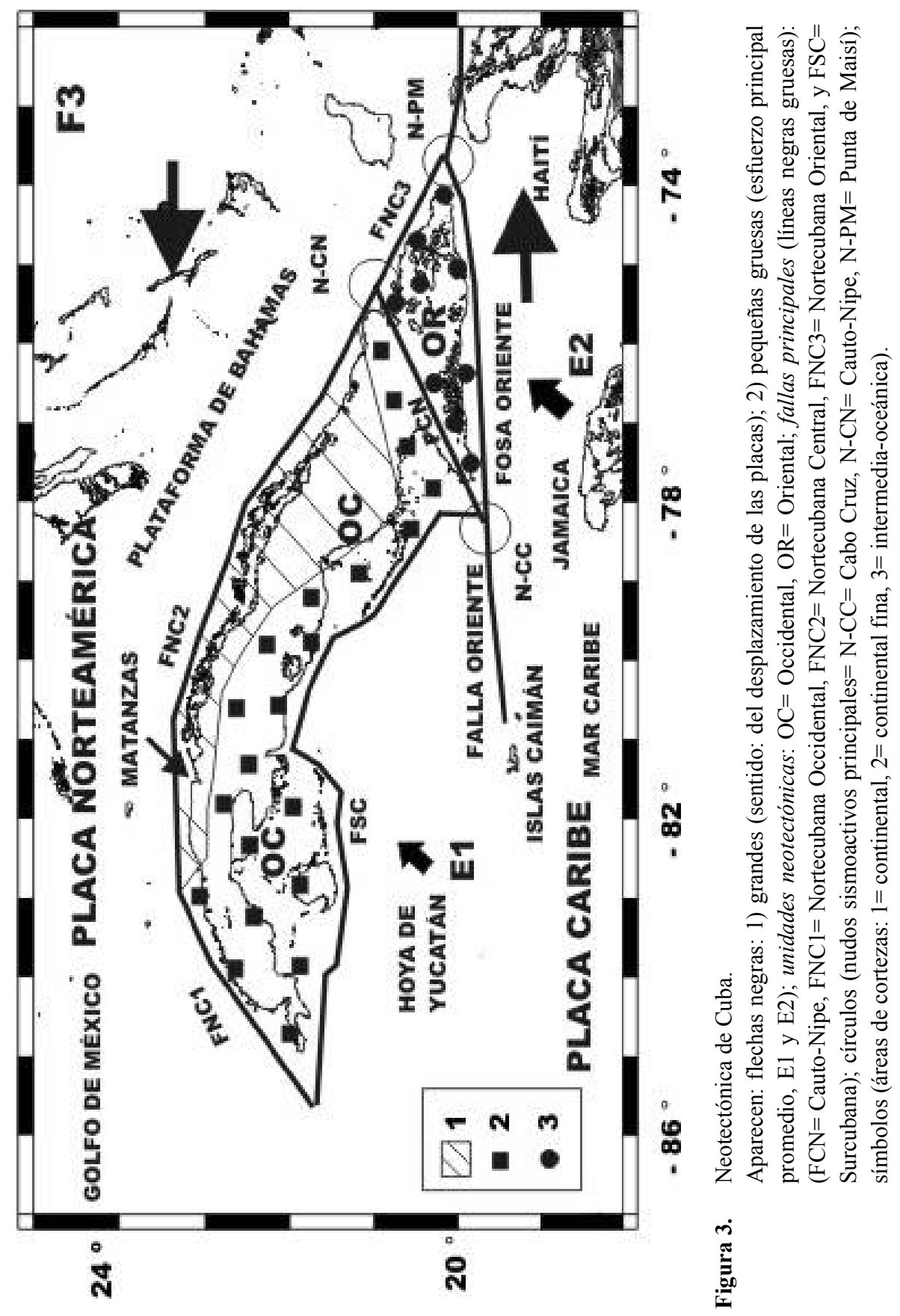


Tabla 1

Datos de las primeras villas cubanas

\begin{tabular}{|c|c|c|}
\hline Denominación inicial (actual) & Región & Fundado (año) \\
\hline Nuestra Señora de la Asunción de Baracoa (Baracoa) & Oriental & 1511 \\
\hline San Salvador de Bayamo (Bayamo) & & 1513 \\
\hline La Habana (Batabanó) & Occidental & 1514 \\
\hline \multicolumn{3}{|l|}{ Sancti Spíritus (Sancti Spíritus) } \\
\hline \multicolumn{3}{|l|}{ La Santísima Trinidad (Trinidad) } \\
\hline San Juan de los Remedios (Remedios) & & 1515 \\
\hline \multicolumn{3}{|l|}{ Nuevitas (Nuevitas) } \\
\hline San Cristóbal de La Habana (Ciudad de La Habana) & & 1519 \\
\hline Santa María de Puerto Príncipe (Camagüey) & & 1528 \\
\hline Minas de Jobabo (Jobabo) & & 1533 \\
\hline Puerto Padre (Puerto Padre) & & 1541 \\
\hline Morón (Morón) & & 1543 \\
\hline Guane (Guane) & & 1600 \\
\hline Esmeralda (Esmeralda) & & 1628 \\
\hline San Diego de los Baños (San Diego de los Baños) & & 1632 \\
\hline Gloriosa Santa Clara (Santa Clara) & & 1689 \\
\hline Matanzas (Matanzas) & & 1693 \\
\hline San Cristóbal de los Pinos (San Cristóbal) & & 1743 \\
\hline Cienfuegos (Cienfuegos) & & $1819(1745)$ \\
\hline Sagua de Tanámo (Sagua de Tanámo) & & 1750 \\
\hline San Isidro de Holguín (Holguín) & & 1751 \\
\hline Tunas de Bayamo (Las Tunas) & & 1752 \\
\hline Güines (Güines) & & 1777 \\
\hline Villa Blanca de Gibara (Gibara) & & 1817 \\
\hline Santa Cruz del Sur (Santa Cruz de Sur) & & 1826 \\
\hline Caibarién (Caibarién) & & 1841 \\
\hline Jagüey Grande (Jagüey Grande) & & 1850 \\
\hline Torriente (Torriente) & & 1863 \\
\hline Ciego de Ávila (Ciego de Ávila) & & 1877 \\
\hline San José de las Lajas (San José de las Lajas) & & 1893 \\
\hline Manatí (Manatí) & & 1912 \\
\hline
\end{tabular}




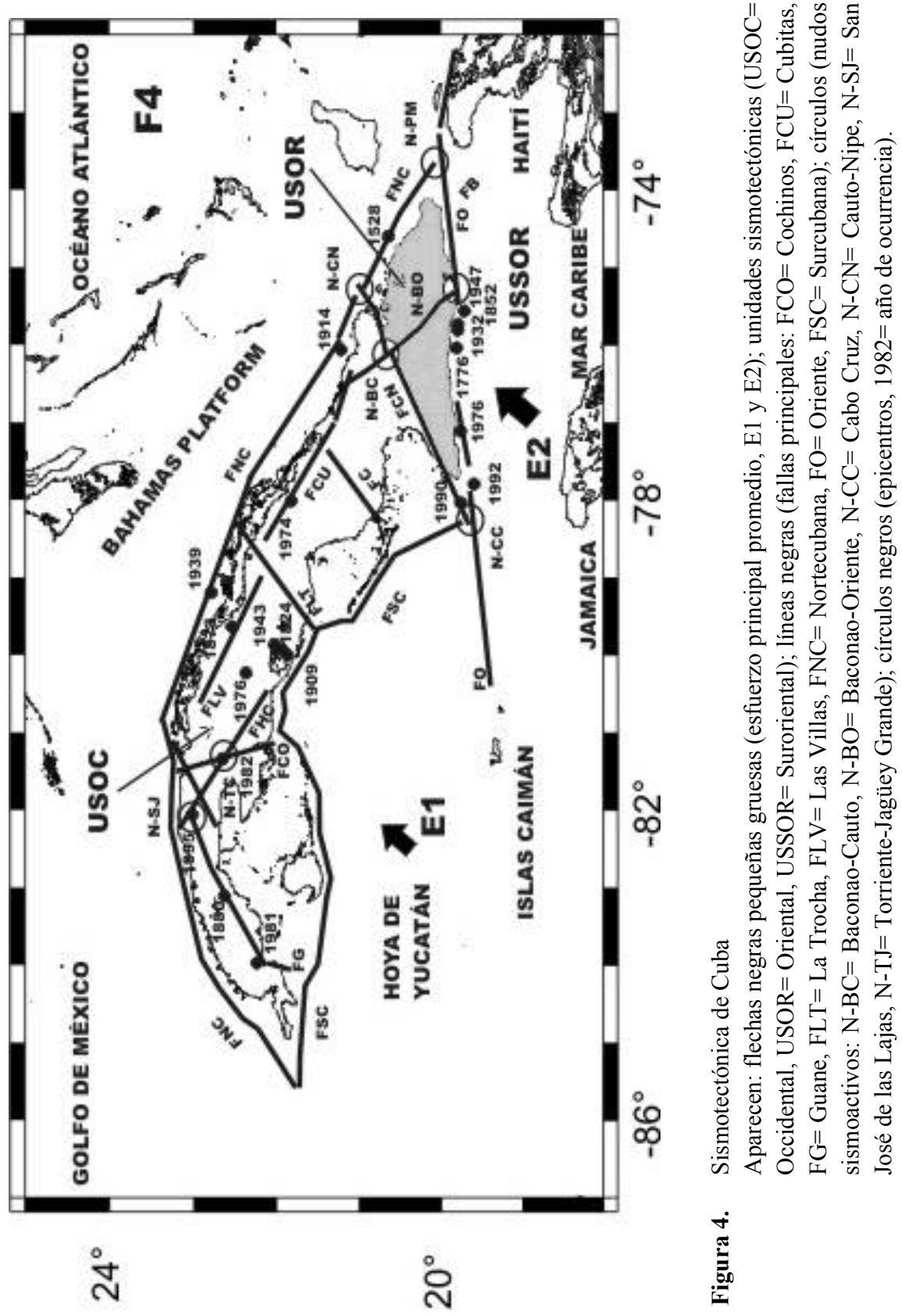




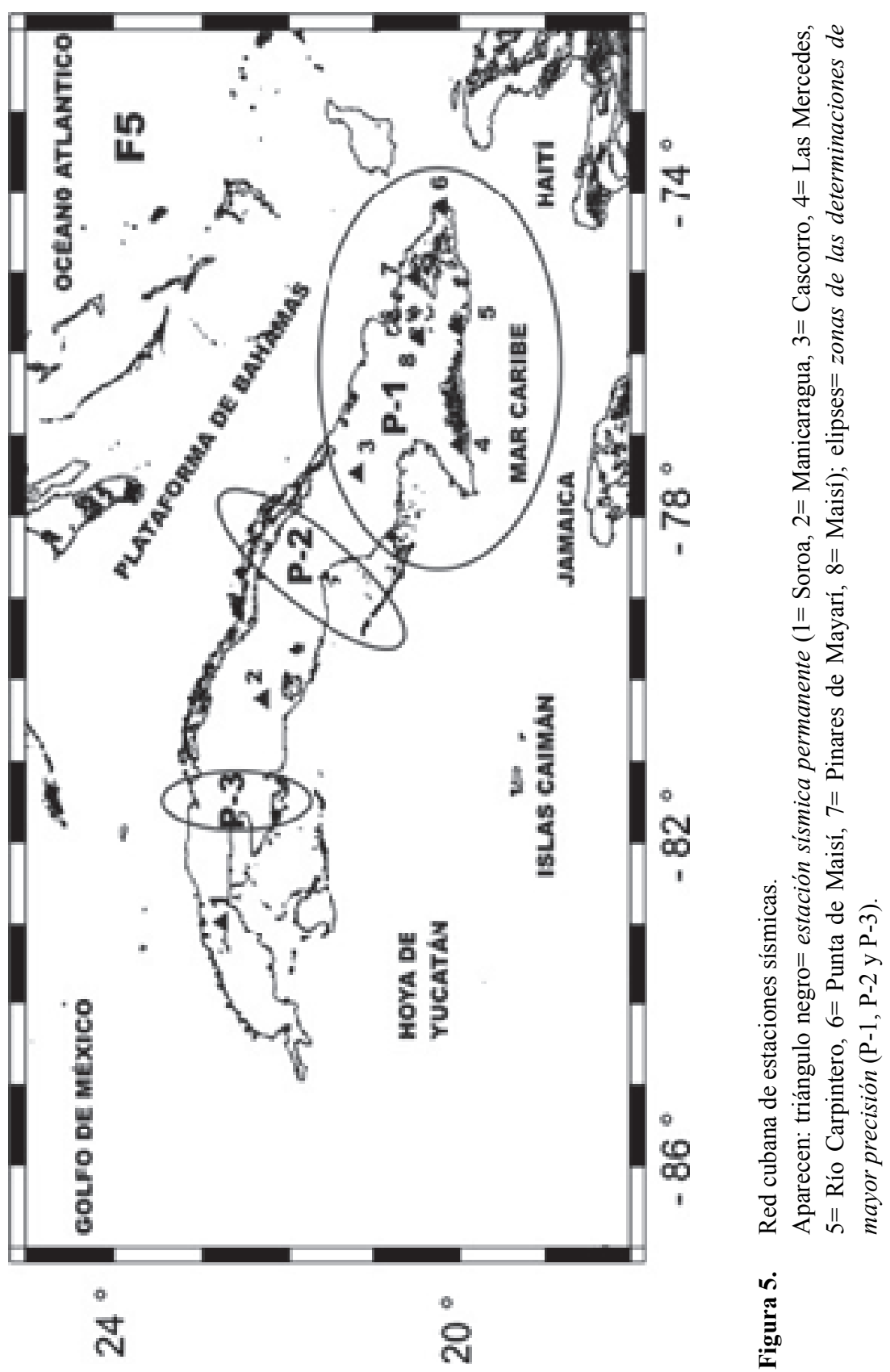




\section{Principales características y datos tectónicos}

Desde nuestro punto de vista, en el trabajo se pueden emplear las siguientes publicaciones para obtener información sobre la tectónica del Caribe: Cotilla y Udías (1999), Erickson et al. (1972), Man y Burke (1984), Mauffret (1996), Prol et al. (1993), Rosencratz (1990), y Rosencratz et al. (1988).

Cuba tiene el plano contemporáneo una figura geométrica de arco cóncavo en la parte norte con el punto de inflexión aproximadamente al este de la bahía de La Habana, y en la zona sur entorno a Batabanó y la bahía de Cochinos (Figura 3). Esto se debe, principalmente, a los esfuerzos paleotectónicos. Aledaño a Cuba hay varias estructuras marinas de importancia que han influido en su evolución neotectónica: Mar Caribe, Fosa de Oriente, Hoya de Yucatán, Golfo de México y Plataforma de Bahamas (Figura 3).

De acuerdo con la tectónica de placas, Cuba se localiza en la placa de Norteamérica e interacciona directamente con la zona de fallas transformantes activas de desplazamiento lateral izquierdo que la separa de la placa Caribe (Figura 2b). La estructura dominante es la fosa Bartlett-Caimán. Ésta es una estructura submarina de tipo pull-apart de $\sim 1,000 \mathrm{~km}$ de longitud a partir de un pequeño centro de generación de corteza oceánica de $110 \mathrm{~km}$ de ancho (Islas Caimán). Ese centro se asume está activo desde el Eoceno medio (velocidad aproximada de expansión $1.5 \mathrm{~cm} / \mathrm{año}$ ), todos estos elementos se enmarcan en una zona límite de placas (ZLP) donde son frecuentes los sismos.

En el Caribe se han producido muchos terremotos fuertes (Figura 2a), la inmensa mayoría de ellos asociados a la ZLP (Cotilla et al., 1997a, b). Ellos conllevan importantes pérdidas humanas y económicas (Cotilla, 2007; Cotilla, 2011a). Consecuentemente, los mayores esfuerzos científicos se han dirigido al estudio de la sismicidad de entreplacas, de hecho la red de estaciones cubanas (Figura 5) está diseñada, fundamentalmente, para esa región (Cotilla, 1993, 2007).

\section{Neotectónica de Cuba Occidental}

En esta parte se hará una breve exposición que se fundamenta principalmente en Cotilla y Álvarez (2001), Cotilla et al. (1991b, 1996, 1998), González et al. (2003), y Prol et al. (1993). Así aseguramos que Cuba es un megabloque emergido y en ascenso diferencial entre sus partes que está incorporado tectónicamente al borde meridional de la placa Norteamérica (Figura 2b). En el megabloque se tienen dos Unidades Neotectónicas (UN): 1) OC, 2) Oriental (OR) (Figura 3), el límite entre ellas es la falla Cauto-Nipe (FCN). Sus particularidades estructurales, complejas y heterogéneas, fueron adquiridas en las dos principales etapas de su desarrollo geológico: orogenia cubana del Eoceno medio y neotectónica en el poseoceno superior. 
Ellas se caracterizan respectivamente por los movimientos compresivos, y los movimientos verticales oscilatorios. A partir de estos últimos se explica la diferenciación y la división en bloques, así como el desarrollo de una cobertura sedimentaria poco deformada que cubre en parte a las estructuras pre neotectónicas. Esa división en bloques se produjo a partir de los límites tectónicos longitudinales y transversales de la etapa pre Eoceno Superior, como zonas de debilidad aprovechadas por las rupturas nuevas, aunque con otro estilo y tendencia, fundamentalmente vertical. La complejidad de la estructura geológica de Cuba se evidencia en cortes geológicos y geofísicos en que aparecen apiladas, mezcladas y dislocadas diferentemente secuencias rocosas de distintas zonas estructurales.

Las principales diferencias neotectónicas entre las dos UN son: 1) altimétricas, 2) orden jerárquico de la red fluvial, 3) afectaciones en los cursos y las divisorias principales de las cuencas fluviales, 4) pendientes fluviales y del relieve, 5) tipos de ríos (encajados), 6) costas afectadas por fallas, 7) densidad de fracturas, 8) cimas puntiagudas, 9) sectores de levantamientos neotectónicos de distintos órdenes, 10) amplitud total de los levantamientos neotectónicos; 11) terrazas marinas, y 12) AS, en todos los casos los mayores valores están en la UNOR (Cotilla et al., 1991b).

La FCN es el límite activo entre las UNOC y UNOR. Esta estructura tiene dos segmentos y contiene tres nudos sismoactivos: 1) Cabo Cruz, 2) San Germán; 3) Banes-Nipe. Esta falla cumple un importante papel en cuanto a geodinámica de Cuba, ya que facilita los desplazamientos laterales y verticales, así como la transmisión de los esfuerzos tectónicos. Además, las principales fallas de Cuba (FCN, falla Nortecubana (FNC) y falla Oriente (FO)) se relacionan con otras del norte del Caribe (Norte de La Española y Swan). En esas zonas se conforman nudos sismoactivos (Figuras 3 y 4 ).

\section{Sismicidad}

Datos y estudios sobre la sismicidad del Caribe y de CO se localizan en: Álvarez et al. (1985, 1990, 1999), Chuy (1989), Chuy y Álvarez (1983), Chuy et al. (1980, 1983a, b, 1984, 1988), Cotilla (1993, 1998a, c, d, 1999, 2003, 2007), Cotilla y Álvarez (1999, 2001), Cotilla y Córdoba (2008, 2010a, b, c, 2011a, b, c), Cotilla et al. (1991a, 1997a, b, 2007), González y Chuy (1983), Frohlich (1982), González et al. (1995), Leroy (1998), Leroy y Mauffret (1996), Montoulieu (1932, 1947, 1968), Orbera et al. (1990), Poey (1855a, b, 1857), Rubio (1982), Salteraín y Legarra (1884), Van Dusen y Dosen (2000), Viñes y Salteraín (1880).

Como primer elemento a considerar en el estudio, es la constatación de que la AS es más fuerte, frecuente y significativa en las islas de La Española (Haití y República Dominicana), Jamaica, y Puerto Rico que en Cuba (Figura 2). Cotilla (2003) y Cotilla y Córdoba (2010b, c) han demostrado con las investigaciones, 
fundamentalmente, en el Archivo de Indias, Sevilla, las sobrestimaciones de la magnitud de varios terremotos históricos de Cuba.

Un resumen del contenido del catálogo de terremotos de $\mathrm{CO}$ aparece en la Tabla 2. Este documento lo prepararon Cotilla y Córdoba (inédito) sobre la base, fundamental, de unos primeros resultados (Cotilla, 1993, 1998a, b, c, d, 1999, 2007, 2011a; Cotilla y Álvarez, 1999, 2001; Cotilla y Córdoba, 2010, 2011a, b; Cotilla y Franzke, 1999; Cotilla et al., 1997a, b). Estos se configuraron con el análisis de los trabajos de: Álvarez et al. (1985, 1990, 1999), Chuy (2003), Chuy et al. (1980, 1983, 1984), González y Chuy (1983), González et al. (1995), Montoulieu (1932, 1947, 1968), Poey (1855, 1855a, 1857), Rubio (1982), Salteraín y Legarra (1884), y Viñes y Salteraín (1880).

El análisis de completitud de nuestro catálogo (Tabla 2) muestra que: 1) de los 921 terremotos son 790 los que tienen información completa de fecha; 2) no es homogéneo en cuanto a intervalos temporales o periodos, destacando el intervalo 1990-1999 con 862 terremotos; 3) la $\mathrm{M}_{\text {máx }}$ es 6.2; 4) las profundidades oscilan entre 10 y $88 \mathrm{~km}$. En la confección se han valorado los terremotos fuertes de las zonas vecinas que han sido perceptibles en Cuba, y consecuentemente están excluidos. Evidentemente, el resultado es una etapa del proceso de confección de un mejor catálogo, pero éste con más de 900 terremotos permite asegurar la existencia de la sismicidad de interior de placa (SIP) del territorio.

Tabla 2

Resumen del catálogo de terremotos de Cuba Occidental

\begin{tabular}{ccccc}
\hline Periodo & Eventos & Mmáx/Mmín & hmáx/hmín $(\mathrm{km})$ & Con fecha completa \\
\hline $1492-1699$ & 3 & $5.0 / 2.6$ & $30 /-$ & - \\
$1700-1799$ & 4 & $3.7 / 3.1$ & $10 /-$ & 2 \\
$1800-1899$ & 35 & $6.2 / 2.5$ & $20 / 10$ & 24 \\
$1900-1999$ & 862 & $6.2 / 0.1$ & $88 / 12$ & 758 \\
$2000-2013$ & 11 & $4.4 / 1.9$ & $33 / 10$ & 6 \\
\hline & 921 & & & 790 \\
\hline
\end{tabular}

La red de estaciones sísmicas permanentes de Cuba aparece representada en la Figura 5. De acuerdo con la cantidad y la distribución espacial de las estaciones es evidente que, la red, aún no puede realizar una detección eficiente en todo el territorio. Así se dan tres elipses de las mejores precisiones (P-1, P-2 y P-3). Para estos estimados se empleó el SIG de Cotilla y Córdoba (2004) suponiendo la ocurrencia de terremotos de diferente magnitud (rangos 2.0-7.0) y con profundidades de $0-30 \mathrm{~km}$. Esto se fundamentó en los datos del periodo instrumental, de la red cuba- 
na. Con esa información se prepararon las Tablas 3 y 4, donde el 75\% de los terremotos tiene magnitudes $\leq 2$ y el $64 \%$ está en profundidades de $1-10 \mathrm{~km}$ (Tabla 4 ). El total de terremotos del catálogo, para el periodo instrumental, es de 498 eventos (Tabla 3) y esto significa como promedio 13 eventos al año. También de la Tabla 3 se extrae que hay dos periodos de gran AS: 1) 1985 (99 eventos); 2) 1988 (78 eventos). Sin embargo, la AS es considerablemente inferior a la existente en la ZLP.

Tabla 3

Detalle de los mejores periodos de registro sísmico (1979-1995)

\begin{tabular}{lccc}
\hline Año & Eventos & Mmáx / Mmín & hmáx $(\mathrm{km})$ \\
\hline 1979 & 6 & 3.1 & 10 \\
1980 & 14 & 3.6 & 50 \\
1981 & 13 & 3.9 & 20 \\
1982 & 16 & 5.0 & 37 \\
1983 & 25 & 3.2 & 50 \\
1984 & 25 & 3.4 & 27 \\
1985 & 99 & 3.3 & 22 \\
1986 & 42 & 3.5 & 20 \\
1987 & 33 & 2.7 & 27 \\
1988 & 78 & 3.2 & 42 \\
1989 & 27 & 2.3 & 27 \\
1990 & 21 & 4.5 & 32 \\
1991 & 17 & 2.7 & 20 \\
1992 & 21 & 4.2 & 40 \\
1993 & 30 & 3.0 & 40 \\
1994 & 14 & 2.3 & 15 \\
1995 & 17 & 3.8 & 30 \\
\hline & 498 & & \\
\hline
\end{tabular}

A partir del catálogo, consideramos que los eventos sísmicos más significativos de CO son los siguientes: 23 de enero de 1880, 28 de febrero de 1914, 15 de agosto de 1939, 30 de julio de 1943, 11 de noviembre de 1970, 26 de julio de 1971, 27 de junio de 1972, 8 de abril de 1974, 16 de diciembre de 1982, 8 de octubre de 1986, 24 de septiembre de 1992, 22 de enero de 1993, y 9 de marzo de 1995 (Tablas 5a, b y Figura 4). Todos ellos están asociados a fallas e identificados como SIP (Tabla 6, Figura 4). También hay otros terremotos de Cuba Oriental que pueden incluirse en este tipo de sismicidad. Ellos son: 17 de enero de 1930, 20 de marzo de 1992, y 28 
de diciembre de 1998 (16 réplicas perceptibles de 500), dos sismos el 5 de enero de 1999, 13 de septiembre de 1999 (37 réplicas perceptibles de 1,340), y 26 de noviembre de 2012.

Tabla 4

Terremotos por rangos de valores de profundidad y magnitud del periodo instrumental (1979-1995)

\begin{tabular}{c|ccccccc}
\hline $\begin{array}{c}h \\
(\mathrm{~km})\end{array}$ & $\begin{array}{c}1-5 \mathrm{~km} \\
(117)\end{array}$ & $\begin{array}{c}5-10 \mathrm{~km} \\
(82)\end{array}$ & $\begin{array}{c}10-15 \mathrm{~km} \\
(47)\end{array}$ & $\begin{array}{c}15-20 \mathrm{~km} \\
(30)\end{array}$ & $\begin{array}{c}20-30 \mathrm{~km} \\
(21)\end{array}$ & $\begin{array}{c}30-50 \mathrm{~km} \\
(12)\end{array}$ & 309 \\
\hline$M$ & $\begin{array}{c}0-2 \\
(366)\end{array}$ & $\begin{array}{c}2-4 \\
(119)\end{array}$ & $>4(5)$ & 490 & & & \\
\hline
\end{tabular}

Tabla 5a

Datos de cinco terremotos de Cuba Occidental

\begin{tabular}{|c|c|c|c|c|c|c|}
\hline \multicolumn{3}{|c|}{ Fecha } & \multirow{2}{*}{$\begin{array}{c}I \\
(M S K)\end{array}$} & \multirow{2}{*}{$\begin{array}{l}M / h \\
(\mathrm{~km}) \\
\end{array}$} & \multirow{2}{*}{$\begin{array}{c}\text { Localidad } \\
\text { (fecha de } \\
\text { fundación) }\end{array}$} & \multirow{2}{*}{$\begin{array}{c}\text { Tiempo en años } \\
\text { desde la fundación / } \\
\text { ocurrencia a } 2013\end{array}$} \\
\hline$D$ & $M$ & $A$ & & & & \\
\hline 23 & enero & 1880 & 8 & $(6.2) / 20$ & $\begin{array}{l}\text { San Cristóbal, Pinar del Río } \\
\text { (1743) }\end{array}$ & $137 / 133$ \\
\hline 28 & febrero & 1914 & 7 & $(6.2) / 20$ & Gibara, Holguín (1751) & $163 / 99$ \\
\hline 15 & agosto & 1939 & 7 & $5.6 / ?$ & $\begin{array}{l}\text { Remedios-Caibarién, Las } \\
\text { Villas (1515) }\end{array}$ & $424 / 74$ \\
\hline 8 & abril & 1974 & 6 & $3.8 / 5$ & $\begin{array}{l}\text { Esmeralda, Ciego de Ávila } \\
\text { (1877) }\end{array}$ & $97 / 39$ \\
\hline 16 & diciembre & 1982 & 6 & $5.0 / 20$ & $\begin{array}{l}\text { Torriente-Jagüey Grande, } \\
\text { Matanzas (1850) }\end{array}$ & $132 / 31$ \\
\hline
\end{tabular}

Tabla 5b

Datos de cinco terremotos de Cuba Occidental

\begin{tabular}{|c|c|c|c|c|c|c|c|c|}
\hline \multicolumn{3}{|c|}{ Fecha } & \multirow[t]{2}{*}{ Isosistas } & \multirow{2}{*}{$\begin{array}{c}\text { Epicentro } \\
\text { en }\end{array}$} & \multirow{2}{*}{$\begin{array}{l}\text { Área } \\
\mathrm{km}^{2}\end{array}$} & \multirow[t]{2}{*}{ Coordenadas } & \multirow[t]{2}{*}{ Réplicas } & \multirow[t]{2}{*}{ Ruptura } \\
\hline$D$ & $M$ & $A$ & & & & & & \\
\hline 23 & enero & 1880 & $\mathrm{Si}$ & Tierra & 40,000 & $\begin{array}{l}22.7 \mathrm{~N} \\
83.0 \mathrm{O}\end{array}$ & 65 & 58 \\
\hline 28 & febrero & 1914 & $\mathrm{Si}$ & Mar & 25,000 & $\begin{array}{l}21.30 \mathrm{~N} \\
76.20 \mathrm{O}\end{array}$ & 9 & 15 \\
\hline 15 & agosto & 1939 & $\mathrm{Si}$ & Mar & 19,000 & $\begin{array}{l}22.50 \mathrm{~N} \\
79.25 \mathrm{O}\end{array}$ & 24 & 53 \\
\hline 8 & abril & 1974 & $\mathrm{Si}$ & Tierra & 8,000 & $\begin{array}{l}21.80 \mathrm{~N} \\
78.05 \mathrm{O}\end{array}$ & 10 & 10 \\
\hline 16 & diciembre & 1982 & $\mathrm{Si}$ & Tierra & 34,000 & $\begin{array}{l}22.61 \mathrm{~N} \\
81.23 \mathrm{O}\end{array}$ & 5 & 41 \\
\hline
\end{tabular}


Tabla 6

Fallas y terremotos de Cuba Occidental (véase Figura 4)

\begin{tabular}{|c|c|c|}
\hline $\begin{array}{l}\text { Denominación } \\
\quad \text { (Siglas) }\end{array}$ & $\begin{array}{c}\text { Mmáx } \\
\text { (Categoria) } \\
\text { Segmentos) } \\
\end{array}$ & Terremotos Asociados (Total) \\
\hline Cubitas (FC) & $4.1(3 / 2)$ & $\begin{array}{l}22 \text { de abril de } 1837 ; 28 \text { de abril de } 1864 ; 25 \text { de mayo } \\
\text { de } 1941 ; 29 \text { de julio de } 1943 ; 1948 ; 16 \text { de marzo de } \\
\text { 1952; } 7 \text { de julio de } 1952 ; 1 \text { de enero de } 1953 ; 25 \text { de } \\
\text { marzo de } 1954 ; 1960 ; 1964 ; 1969 ; 27 \text { de junio de } \\
\text { 1972; } 8 \text { de abril de } 1974 ; 8 \text { de octubre de } 1986(15)\end{array}$ \\
\hline Camagüey (FCA) & $3.0(4 / 1)$ & $\begin{array}{l}\text { 1770; } 12 \text { de abril de } 1776 ; 28 \text { de abril de } 1846 \text {; octu- } \\
\text { bre de } 1846 ; 7 \text { de marzo de } 1858 ; 23 \text { de noviembre de } \\
\text { 1949; } 10 \text { de noviembre de } 1952 ; \text { septiembre de } 1955 ; \\
\text { diciembre de } 1966 ; 16 \text { de diciembre de } 1954 \text { (10) }\end{array}$ \\
\hline Cauto-Nipe (FCN) & $6.6(2 / 2)$ & $\begin{array}{l}18 \text { de octubre de } 1551 \text {; octubre de } 1624 ; 26 \text { de no- } \\
\text { viembre de } 1856 ; 20 \text { de enero de } 1858 ; 3 \text { de agosto de } \\
1926 ; 16 \text { de abril de } 1987 ; 25 \text { de abril de } 1987 ; 26 \text { de } \\
\text { agosto de } 1990 ; 25 \text { de mayo de } 1992 ; 27 \text { de junio de } \\
1995 \text { (10) }\end{array}$ \\
\hline Cochinos (FCH) & $5.0(3 / 2)$ & $\begin{array}{l}\text { 1903; enero de } 1927 ; 5 \text { de junio de } 1928 ; 27 \text { de marzo } \\
\text { de } 1964 ; 1974 ; 16 \text { de diciembre de } 1982 \text { (6) }\end{array}$ \\
\hline Guane (FG) & $6.2(2 / 3)$ & $\begin{array}{l}23 \text { de enero de } 1880 ; 31 \text { de agosto de } 1886 ; 23 \text { de } \\
\text { septiembre de } 1921 ; 9 \text { de marzo de } 1936 ; 20 \text { de di- } \\
\text { ciembre de } 1937 ; 15 \text { de febrero de } 1939 ; 24 \text { de sep- } \\
\text { tiembre de } 1939 ; 9 \text { de marzo de } 1955 ; 11 \text { de } \\
\text { septiembre de } 1957 ; 1958 ; 1964 ; 1974 ; 10 \text { de marzo } \\
\text { de } 1976 ; 15 \text { de marzo de } 1976 ; 1978 ; 1982 ; \text { septiem- } \\
\text { bre de } 1988 \text { (17) }\end{array}$ \\
\hline $\begin{array}{l}\text { Habana-Cienfuegos } \\
\text { (FHC) }\end{array}$ & $5.0(3 / 4)$ & $\begin{array}{l}\text { 1693; } 1810 ; 1835 ; 8 \text { de marzo de } 1843 ; 21 \text { de febrero } \\
\text { de } 1843 ; 1844 ; 1852 ; 1854 ; 4 \text { de octubre de } 1859 ; \\
\text { diciembre de } 1862 ; 25 \text { de marzo de } 1868 ; 1880 ; 15 \text { de } \\
\text { abril de } 1907 ; 1941 ; 18 \text { de diciembre de } 1942 ; 11 \text { de } \\
\text { septiembre de } 1947 ; 10 \text { de febrero de } 1970 ; 16 \text { de } \\
\text { diciembre de } 1982 ; 9 \text { de marzo de } 1995(19)\end{array}$ \\
\hline Hicacos $(\mathrm{FH})$ & $3.0(3 / 3)$ & $\begin{array}{l}\text { 1812; } 5 \text { de marzo de } 1843 ; 1852 ; 1854 ; 10 \text { de sep- } \\
\text { tiembre de } 1854 ; 1880 ; 27 \text { de mayo de } 1914 ; 28 \text { de } \\
\text { mayo de } 1914 ; 27 \text { de abril de } 1974 ; 1978 \text { (10) }\end{array}$ \\
\hline La Trocha (FLT) & $4.6(4 / 1)$ & $\begin{array}{l}30 \text { de julio de } 1943 ; 11 \text { de noviembre de 1970; } 26 \text { de } \\
\text { julio de } 1971 \text { (3) }\end{array}$ \\
\hline Las Villas (FLV) & $4.5(3 / 2)$ & $\begin{array}{l}15 \text { de agosto de } 1939 ; 3 \text { de febrero de } 1952 ; 1 \text { de } \\
\text { enero de } 1953 ; 25 \text { de mayo de } 1960 ; 22 \text { de enero de } \\
1983 ; 4 \text { de enero de } 1988 \text { (6) }\end{array}$ \\
\hline Nortecubana (FNC) & $6.2(2 / 3)$ & $\begin{array}{l}28 \text { de febrero de } 1914 ; 15 \text { de agosto de } 1939 ; 25 \text { de } \\
\text { mayo de } 1960 ; 18 \text { de diciembre de } 1986 ; 5 \text { de enero } \\
\text { de } 1990 ; \text { (dos) } 5 \text { de enero de } 1992 ; 20 \text { de marzo de } \\
1992 ; 24 \text { de septiembre de } 1992 ; 28 \text { de diciembre de } \\
1998 \text { (10) }\end{array}$ \\
\hline Surcubana (FSC) & $(5 /-)$ & $\begin{array}{l}28 \text { de abril de } 1846 ; 10 \text { de septiembre de } 1846 ; 18 \text { de } \\
\text { abril de } 1847 ; 24 \text { de enero de } 1909 ; \text { julio de } 1943 ; 30 \\
\text { de julio de } 1943 ; 28 \text { de abril de } 1946 ; 23 \text { de noviem- } \\
\text { bre de } 1949 \text { (8) }\end{array}$ \\
\hline
\end{tabular}


Una comparación por intensidad sísmica para cuatro terremotos fuertes de CO, así como la información sobre las escasas investigaciones de campo se encuentra en la Tabla 7. En la Tabla 8 aparecen las distancias y los tiempos de ocurrencia entre los cinco terremotos de mayor magnitud de CO. De estas tablas se puede colegir que los eventos: 1) están mucho más espaciados que los de la ZLP; 2) poseen tiempos de ocurrencia mayores que los de la ZLP.

$\mathrm{El}$ autor ha utilizado los datos de terremotos registrados con estaciones sísmicas portátiles de tipo Tierra, de una campaña realizada por especialistas rusos en los años 1974-1975 (Sherbakova et al., 1977) (Figura 6a). En este sentido hay siete epicentros en las inmediaciones de Cuba Central (Trinidad-Sancti-SpíritusJatibónico) y 52 en Esmeralda. En ambos entornos se han producido sismos perceptibles, entre ellos los de 1970 (M=3.2 en Sancti Spíritus) y 1971 (M=3.3 en Jatibónico), y en Esmeralda citamos dos: $1972(\mathrm{M}=3.7)$ y $1974(\mathrm{M}=3.8)$. Otros tres terremotos de la campaña Tierra se corresponden con la falla Surcubana, por lo que confirman su actividad.

Tabla7

Datos de los cuatro terremotos más fuertes de Cuba Occidental (I $>5$ e I $>7$, MSK)

\begin{tabular}{lcccc}
\hline \multicolumn{1}{c}{ Terremoto } & $\begin{array}{c}I \geq 5(\mathrm{MSK}) \\
\text { cantidad }\end{array}$ & $\begin{array}{c}I \geq 7 \text { (MSK) } \\
\text { cantidad }\end{array}$ & $\begin{array}{c}\text { hmáx } \\
(\mathrm{km})\end{array}$ & $\begin{array}{c}\text { Trabajos de } \\
\text { campo }\end{array}$ \\
\hline San Cristóbal & 2 & 2 & 20 & SI \\
Gibara & 1 & 1 & 10 & - \\
Remedios-Caibarién & 2 & 1 & 15 & - \\
Torriente-Jagüey Grande & 1 & - & 30 & SI \\
\hline & 6 & 4 & & \\
\hline
\end{tabular}

Tabla 8

Distancias y tiempos entre los cuatro terremotos más fuertes de Cuba Occidental

\begin{tabular}{ccc}
\hline $\begin{array}{c}\text { Localidad 1 / } \\
\text { Localidad 2 }\end{array}$ & Distancia $(\mathrm{km})$ & Tiempo (años) \\
\hline SC / G & 825 & 34 \\
SC / TJG & 200 & 102 \\
SC / RC & 400 & 59 \\
G / TJG & 620 & 68 \\
G / RC & 450 & 25 \\
TJG / RC & 175 & 43 \\
\hline
\end{tabular}

Nota: G: Gibara; RC: Remedios-Caibarién; SC: San Cristóbal; TJG: Torriente-Jagüey Grande. 
La comparación espacial, por distancia mínima y su error asociado, calculado con el SIG de Córdoba y Cotilla (2004) y empleando las Figuras 4 y 6a para 13 terremotos de $\mathrm{CO}$ muestra un $78 \%$ de coincidencia. Y la comparación para el conjunto de fallas resulta ser del $71 \%$ de coincidencia.

La Figura 6b tiene los epicentros determinados (1975-1990) con la estación de Soroa, Pinar del Río. Aunque es evidente que la fiabilidad es baja, es posible comprobar que esos eventos se asocian por completo al entorno de la mencionada estación y por consiguiente son de tipo interior de placas. Por otra parte, la sismicidad de Cuba Oriental y Cuba Suroriental (1979-1990) con los datos de la red de estaciones sísmicas cubanas, de entonces, aparece en la Figura 6c. En esta ocasión es factible apreciar: 1) una considerable cantidad de epicentros en todo el borde suroriental y en particular al sur de la Sierra Maestra; 2) 10 grupos de epicentros; 3) que la sismicidad es aparentemente mayor en la parte norte-occidental (al oeste de la FCN) que en la parte norte-oriental (este de la FCN); 4) muchos epicentros en la región insular de Cuba. Con estos dos últimos puntos se puede asegurar que hay SIP.

\section{Tabla 9}

Otros terremotos de la parte Oriental de Cuba

\begin{tabular}{ll}
\hline \multicolumn{1}{c}{ Localidad } & \multicolumn{1}{c}{ Fecha (Magnitud) } \\
\hline Caibarién & 15 de agosto de 2012 (2.4) \\
Centro de Oriente & Marzo de 2003 (2.1) \\
Cumanayagua & 8 de agosto de 1996 (3.8) \\
Encrucijada & 20 de noviembre de 2012 (3.3) \\
Esmeralda & 26 de noviembre de 2012 (2.5) \\
Gibara & 19 de noviembre de 2001 (4.2); febrero de 2003 (1.9); marzo de 2003 \\
& $(2.2) ; 10$ de junio de 2007 (4.0) \\
Moa & 28 de diciembre de 1998 (5.6); 30 de diciembre de 1998 (3.6); 5 de \\
& enero de 1999 (4.7); 5 de enero de 1999 (4.5); 5 de julio de 1999 \\
& (3.7) \\
Nuevitas & 13 de octubre de 2003 (4.4) \\
\hline
\end{tabular}

Retomando el punto (3) comprobamos con la Tabla 9 que algunos terremotos importantes fueron registrados, por la red cubana, durante el periodo 1988-2012 en el norte de Cuba Oriental sobre la FNC y al este de la FCN (Tabla 9). Esto fue previsto con argumentos de neotectónica y microtectónica por Cotilla (1993), entonces se aseguró, ante la Administración, que excluir esa zona del programa de investigaciones de la Central Electronuclear (1979-1989) que se planificaba en Holguín era un error de pérdida de objetivo. El autor dijo entonces que la FNC, entorno a Nipe, estaba segmentada, y que la parte oriental era la más activa. En la actualidad soste- 
nemos que hay una importante influencia del tensor de esfuerzos de la interacción de placas Caribe-Norteamérica en ambos segmentos de la FNC.
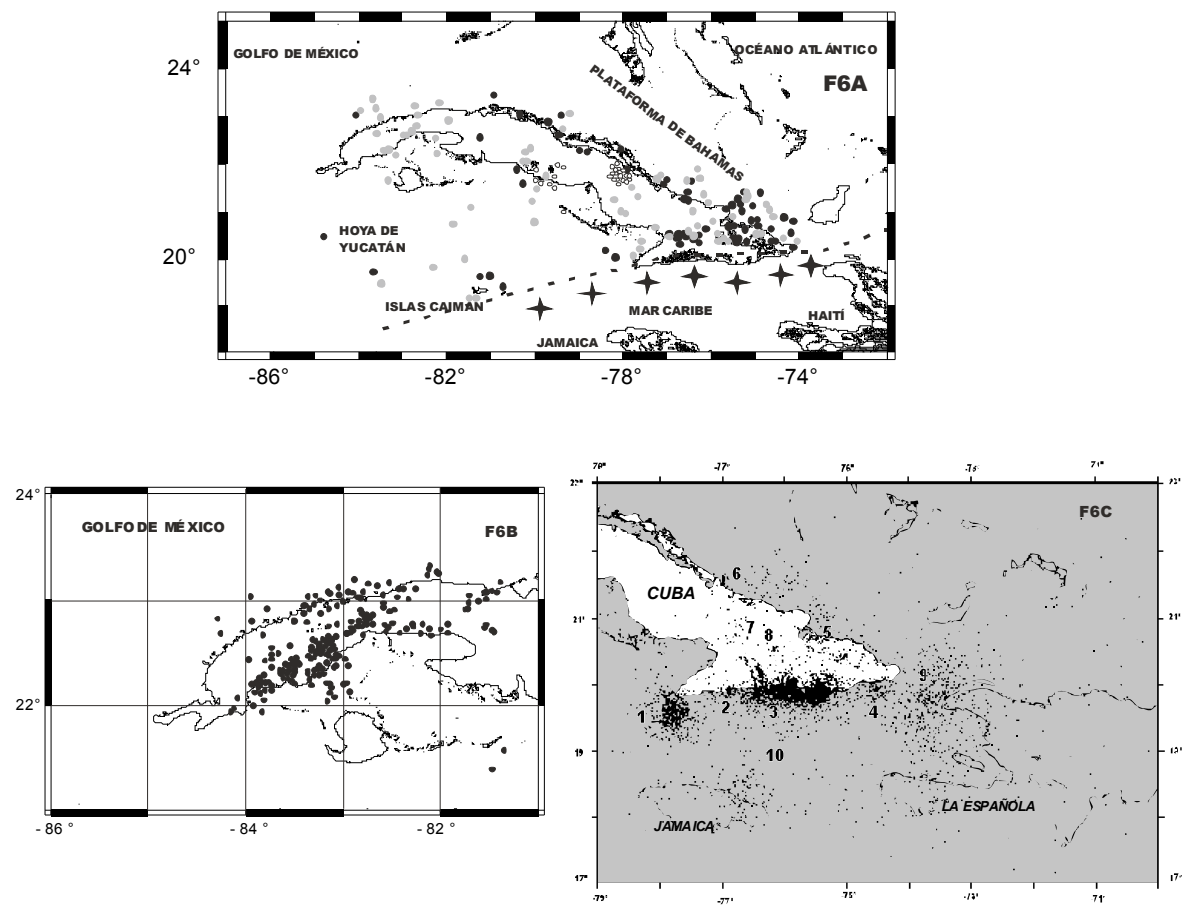

Figura 6a. Sismicidad de Cuba a partir de la red cubana (1979-2000) y los equipos "Tierra" (1974-1975).

Selección de epicentros: 1) red cubana (círculos negros=1979-1990 y círculos grises $=1990-2000 ; 2)$ "Tierra" (círculos en blanco= 1974-1975); línea negra discontinua y estrellas negras $=$ zona no incluida.

Figura 6b. Sismicidad de Pinar del Río (estación Soroa).

Selección de epicentros (círculos negros=1979-1990).

Figura 6c. Sismicidad de Cuba Oriental y Cuba Suroriental (red cubana 1979-1990).

Selección de epicentros (círculos negros); Agrupaciones de epicentros $1=\mathrm{Cabo}$ Cruz, 2= Pilón, 3= Santiago-Baconao, 4= Imías-San Antonio, 5= Nipe, 6= Puerto Padre, $7=$ Tunas-Jobabo, $8=$ San Germán, 9= Maisí, 10= Jamaica.

Otras zonas aledañas a Cuba, también con SIP, son: 1) el Golfo de México: 7 de julio de $1852(\mathrm{M}=7.5), 6$ de mayo de 1905, 14 de diciembre de 2004, 20 de diciembre de $2004(\mathrm{M}=6.6), 10$ de febrero de $2006(\mathrm{M}=5.2), 10$ de septiembre de $2006(\mathrm{M}=5.8), 29$ de octubre de $2009(\mathrm{M}=5.5), 8$ de octubre de $2012(\mathrm{M}=5.7)$, y 26 de abril de $2011(\mathrm{M}=5.6)$; 2) Bahamas donde se localizan terremotos por la red 
cubana (Figura 6a), por ejemplo del 22 de febrero de $1992(\mathrm{M}=3.2)$; 3) Hoya de Yucatán hay al menos tres terremotos registrados con la red cubana (Figura 6a); 4) el interior del Mar Caribe, como ejemplo uno del 3 de abril de 2007 (M=3.6).

Tabla 10a

Datos sobre algunos de los terremotos más importantes de la zona de entre placas (Cuba suroriental)

\begin{tabular}{cccc}
\hline Fecha & $M / I(M S K) / h(\mathrm{~km})$ & Coordenadas & Ruptura $(\mathrm{km})$ \\
\hline $\begin{array}{c}11 \text { de junio de } \\
1766\end{array}$ & $6.8 / 9 / 25$ & $19.9 \mathrm{~N} / 76.1 \mathrm{O}$ & 85 \\
$\begin{array}{c}20 \text { de agosto } \\
\text { de } 1852\end{array}$ & $6.4 / 8 / 30$ & $19.75 \mathrm{~N} / 75.33 \mathrm{O}$ & 75 \\
$\begin{array}{c}03 \text { de febrero } \\
\text { de } 1932\end{array}$ & $6.75 / 8 / 35-40$ & $19.8 \mathrm{~N} 75.8 \mathrm{O}$ & 65 \\
$\begin{array}{c}07 \text { de agosto } \\
\text { de } 1947\end{array}$ & $6.7 / 7 / 30$ & $19.54 \mathrm{~N} 75.3 \mathrm{O}$ & 49 \\
$\begin{array}{c}19 \text { de febrero } \\
\text { de } 1976\end{array}$ & $5.7 / 8 / 15$ & $19.87 \mathrm{~N} 76.87 \mathrm{O}$ & 55 \\
$\begin{array}{c}26 \text { de agosto } \\
\text { de } 1990\end{array}$ & $5.9 / 8 / 10$ & $19.6 \mathrm{~N} 77.87 \mathrm{O}$ & 60 \\
$\begin{array}{c}25 \text { de mayo de } \\
1992\end{array}$ & $6.9 / 15 / 23$ & $19.61 \mathrm{~N} 77.87 \mathrm{O}$ & 65 \\
\hline
\end{tabular}

Tabla 10b

Datos sobre los tres terremotos más importantes de Cuba Suroriental

\begin{tabular}{|c|c|c|c|}
\hline \multirow[b]{2}{*}{ Parámetros } & \multicolumn{3}{|c|}{ Terremotos } \\
\hline & 1766 & 1852 & 1932 \\
\hline Fecha (día y mes) & 11.06 & 20.08 & 03.02 \\
\hline Magnitud & 6.8 & 6.4 & 6.75 \\
\hline Imáx (MSK) & 9 & 8 & 8 \\
\hline Profundidad (km) & 25 & 30 & $35-40$ \\
\hline Coordenadas & $19.9 \mathrm{~N} / 76.1 \mathrm{O}$ & $19.75 \mathrm{~N} / 75.32 \mathrm{O}$ & $19.75 \mathrm{~N} / 75.58 \mathrm{O}$ \\
\hline Muertos / Heridos & $34-40 / 700$ & $2 / 200$ & $14 / 300$ \\
\hline $\begin{array}{l}\text { Área perceptibilidad }\left(10^{3}\right. \\
\left.\mathrm{km}^{2}\right)\end{array}$ & $\sim 110$ & $\sim 80$ & $\sim 90$ \\
\hline Epicentro en el mar & SI & SI & SI \\
\hline
\end{tabular}

Los siete terremotos importantes de la ZLP septentrional del Caribe (segmento de Cuba Suroriental) están en las Tablas 10a, 10b y Figura 4. Estos eventos se encuentran en la zona de mayor densidad epicentral indicada en la Figura 6c. Cotilla (2007) asegura que las cifras de fallecidos por terremotos en esta parte de Cuba (1514-2013) no alcanza el centenar ( 20). Esa cifra es muy baja de compararla con 
las conocidas de las islas vecinas. Como ejemplo está la Tabla 10b que recoge las cifras de muertos $(\sim 56)$ con sólo tres de los más fuertes terremotos de Cuba Suroriental. Viñes y Gutiérrez Lanza, ya citados "las exageraciones en pérdidas económicas y humanas, en los casos de terremotos históricos de Cuba", más recientemente, Montoulieu (1932) expuso un punto de vista similar durante las investigaciones de campo con el terremoto de 1932. Cotilla (2003) y Cotilla y Córdoba $(2008,2010 b, c)$ comprobaron lo anterior en las búsquedas realizadas en el Archivo de Indias, Sevilla.

Los pares de Tablas 5a, 5b, 10a y 10b muestran los datos de los terremotos más importantes de $\mathrm{CO}$ y Cuba Suroriental, respectivamente. En este sentido se ve que las magnitudes, las áreas de perceptibilidad, y las dimensiones de ruptura estimadas son siempre mayores en la región Suroriental. Esto se corresponde con lo esperado de una zona de interacción de placas.

En la zona de Islas Caimán hay varios terremotos fuertes que han afectado a Cuba, entre ellos está el del 7 de julio de $1852(\mathrm{Ms}=7.5)$; 14 de diciembre de 2004 $(\mathrm{M}=6.8)$. Pero estos son de límite de placas, y se relacionan directamente con una cuenca de pull-apart o graben con forma de rombo y estructuras de bloques tipo horst, están asociadas con sistemas de fallas de deslizamiento lateral, como la FO. Muchas cuencas de pull-apart y sistemas de bloques de presión (pressure ridge), con dimensiones varias, se han estudiado en diferentes zonas, pero siempre hay sismicidad asociada. Las dimensiones de estas cuencas, en el plano varían, pero las más frecuentes son: $\operatorname{largo}=0.5 \mathrm{~m}-80 \mathrm{~km}$, ancho $=0.2 \mathrm{~m}-40 \mathrm{~km}$, como es el caso de Cauto.

Tabla 11

Datos del catálogo de Álvarez et al. (1999) $\left(\mathrm{h} \leq 30 \mathrm{~km} ; 1^{\circ} \mathrm{N}-24^{\circ} \mathrm{O} /\right.$ $\left.^{\circ} 7^{\circ} \mathrm{O}-86^{\circ} \mathrm{O}\right)$

\begin{tabular}{cccccccc}
\hline & & & & & \multicolumn{3}{c}{ Sin datos de } \\
\cline { 6 - 8 } Periodo & $\geq 5$ & $\geq 6$ & $\geq 7$ & $\geq 8$ & $D$ & $M$ & $D M$ \\
\hline $1502-1580$ & 7 & 4 & 2 & - & 4 & 5 & 4 \\
$1615-1693$ & 13 & 8 & 4 & - & 6 & 7 & 6 \\
$1701-1793$ & 22 & 11 & 3 & - & 8 & 12 & 8 \\
$1800-1899$ & 36 & 14 & 7 & 1 & 27 & 55 & 27 \\
$1900-1995$ & 163 & 33 & 12 & 1 & 82 & 127 & 82 \\
\hline & 241 & 70 & 28 & 2 & 127 & 206 & 127 \\
\hline
\end{tabular}

Nota: $\mathrm{D}=$ día, $\mathrm{M}=$ mes, $\mathrm{DM}=$ día y mes.

Con la Tabla 11 es posible visualizar una comparación entre los catálogos del autor (aquí empleado) y el de Álvarez et al. (1999), este último incluye terremotos para una región mucho mayor $\left(16^{\circ} \mathrm{N}-24^{\circ} \mathrm{N}, 67^{\circ} \mathrm{O}-86^{\circ} \mathrm{O}\right)$. Los rangos temporales son diferentes: 1) del autor (1492-1995); 2) Álvarez et al. (1999) (1502-1995). Y en 
el análisis se ha acotado la comparación para profundidades focales $\mathrm{de} h \leq 30 \mathrm{~km}$, esto significa que nuestra elección es adecuada.

\section{Discusión sismotectónica}

Es conocido que la Tierra está dividida en dos grandes dominios: 1) placas prácticamente rígidas; 2) zonas límites de placas con importantes deformaciones. Así, la teoría sobre la tectónica y sismicidad de zonas de interior de placas se puede localizar entre otros en: Wdowinski (1998) y Scholtz et al. (1986). La actividad de las fallas en las regiones de interior de placa se caracteriza por: 1) su bajo nivel de AS; 2) estar cubiertas de sedimentos; 3) la no relación directa y evidente con los límites de placas, a pesar de que muchas de ellas son antiguas estructuras de ese tipo; 4) requerir de estudios geomorfológico-estructurales para delimitar su existencia y actividad contemporánea. Un punto de vista interesante sobre la no homogeneidad de la Tectónica de Placas está en Smoot (1997), sostiene que las alineaciones de elevaciones, fracturas, etc., en zonas de subducción no se corresponden con la mencionada teoría. Sin embargo, estas apreciaciones no modifican la idea de considerar la existencia de SIP.

Es bien conocido que los terremotos son un gran peligro natural para las naciones. Muchas situaciones dramáticas se han visto en grandes e importantes ciudades como: 1) Bucarest-Rumania el 4 de marzo de 1977 (M=7.2 y 1,500 muertos); 2) Campania-Italia el 23 de noviembre de 1980 ( $\mathrm{M}=6.5$ y 3,000 muertos); 3) Atenas el 24 de febrero de 1981 ( $\mathrm{M}=6.8$ y 16 muertos) y 7 de septiembre de $1999(\mathrm{M}=6.0$ y 143 muertos); y 4) el 17 de agosto de 1999 en Izmit-Turquía (M=7.6 y 17,000 muertos). En todas ellas las pérdidas humanas y económicas han sido muy grandes, del 3-7\% del PIB. Otro ejemplo de terremoto fuerte y destructor se encuentra en el SO de Bulgaria (Meyer et al., 2002). El terremoto se produjo el 4 de abril de 1904, $\mathrm{M}_{\mathrm{s}}=7.8, \mathrm{I}_{\text {máx }}=10$ (MSK). En esta ocasión no existía un buen estimado de peligrosidad sísmica, ya que las fallas se consideraban ocultas. Aquí caben las siguientes preguntas: 1) ¿En qué tipo de zonas se produjeron todos esos terremotos, interior de placa o entreplacas?; 2) ¿Cuál fue el mecanismo predominante, interacción directa o transmisión de esfuerzos desde la ZLP? Estos son ejemplos de acción directa de las placas. También de una zona de colisión de placas, tenemos las muy importantes observaciones de Balassanian et al. (1995) sobre las investigaciones del terremoto de Spitak, Armenia (7 de diciembre de 1988) que se pueden extender a los terremotos mencionados. Ellas indican varias cosas de interés en Sismotectónica, entre ellas la ruptura múltiple de segmentos de fallas. Ese terremoto $\left(\mathrm{M}_{\mathrm{s}}=6.9, \mathrm{~h}=10 \mathrm{~km}, \mathrm{I}_{\text {máx }}=\right.$ $10 \mathrm{MSK}$, área de ruptura de $\sim 300 \mathrm{~km}^{2}$ ) produjo la destrucción total de tres ciudades, entre ellas Spitak. Las pérdidas humanas fueron $\sim 25,000$ y las económicas superaron los 16 billones de dólares. El terremoto y sus réplicas ocurrieron en una zona de 
colisión de placas, donde la sismicidad no era desconocida, y los especialistas estimaron, para el diseño de las edificaciones, una $\mathrm{I}_{\text {máx }}=7$ (MSK). Ese error por defecto, evidentemente, facilitó la catástrofe. Luego, para el caso de una zona de interior de placas resultan aún más difíciles los diagnósticos de: magnitud-intensidad, localización y tiempo.

En el Caribe también se han producido grandes pérdidas humanas por causa de terremotos de entreplacas. Entre ellos están: Jamaica: 1) 7 de junio de 1692 (2,000 muertos), 2) 14 de enero de 1907 (1,000 muertos); Guatemala: 1) 19 de abril de 1902 (2,000 muertos), 2) 4 de febrero de 1976 (23,000 muertos); Costa Rica 22 de abril de 1991 (47 muertos); y Colombia 25 de enero de 1999 (1,185 muertos). Y en particular Russo y Vilasenor (1995) observaron que la sismicidad reciente es relativamente baja y poco profunda en el entorno del área epicentral del terremoto de 4 de agosto de 1946 (Ms= 7.8), al N de República Dominicana, Península de Samaná (Figura 4). Ese evento, de tipo entreplacas, tuvo 63 réplicas con profundidades de 70-130m, 100 muertos y un tsunami. La explicación es que la liberación energética del terremoto redujo la tensión de la región.

De otra parte, existen muchos estudios sobre la relación entre las fallas y la sismicidad (Evison, 1963; Griggs y Handin, 1960; Mogi, 1967, Orowan, 1960). Abers (1991), y Goodluck y Ferrill (1998) han propuesto que los sistemas de fallas de tipo normal, diferentemente ajustadas en su perfil por planos de deslizamiento paralelos pueden generar series de terremotos. Esos sistemas de fallas lístricas están relacionados con un campo de esfuerzos de tipo extensional, aunque el contexto regional puede ser diferente (esfuerzos compresivos o transcompresivos) (Cotilla, 1993; Cotilla y Córdoba, 2003).

Las propuestas de Wyss (1979), Wells y Coppersmith (1984), y Schwartz y Coppersmith (1986), para la relación entre longitud de las fallas y la magnitud de los terremotos, fueron aplicadas en el MS de Cuba (Cotilla, 1993). También se consideró que las fallas están segmentadas (Cotilla et al., 2007).

Wallace (1984) determinó que la concentración de fallas en determinadas áreas estaba relacionada con la ocurrencia de terremotos y que éstos dependían de la taxonomía y jerarquía regionales del territorio a manera de pulsos. Esto se evidenciaba a partir del diferente comportamiento sísmico en las provincias y zonas de menor rango, pero entrelazadas por la actividad en la corteza y el manto superior. Ese fue el argumento de: 1) Cotilla et al. (1991a) para establecer las tres Unidades Sismotectónicas (US): Occidental (OC), Oriental (OR), y Suroriental (SOR) (Figura 4); 2) Cotilla et al. (1998) para considerar la división del territorio de Cuba Oriental en tres celdas geodinámicas. Es decir, que hay fragmentación de las zonas sísmicas en Cuba.

En este sentido, diversos autores han estudiado las zonas de intersección de fallas como regiones sísmicamente activas (Zhidkov et al., 1975). Arsovsky y Had- 
zievsky (1970) determinaron para Macedonia que: 1) los focos de terremotos fuertes están en los cruces o intersecciones de las dislocaciones; 2) las áreas de mayor sismicidad son esas intersecciones; 3 ) muchas áreas donde están bien expresadas las fallas demuestran AS, pero no siempre es así. Spotilla y Anderson (2004) establecieron, para una zona de California, una diferenciación de fallas activas a partir del análisis de estructuras transversales a ellas. De acuerdo con Cotilla (1993), Cotilla y Álvarez (2001), Cotilla y Franzke (1999), y Cotilla et al. (1991a, 1996, 2007); los terremotos en Cuba se localizan en fallas e intersecciones de fallas. Y en la Figura 6 b de Moreno Toirán (2002) el terremoto de Moa (28 de diciembre de 1998) está en la intersección de las FNC y FCN. Ese es el nudo N3 de Cotilla (1993) (Figuras 4 y 6c). Además, de observar la Figura 4 se aprecia que, en la parte Oriental, hay cinco nudos sismoactivos y en $\mathrm{CO}$ hay dos. Ellos permiten justificar la cinemática de desplazamiento lateral de la región y la rotación de bloques a menor escala taxonómica.

Teisseyre (1970) demostró que la acumulación de esfuerzos en una determinada región conlleva a la posterior liberación energética, por lo que el estudio complejo de ese entorno puede ayudar en el pronóstico de terremotos. Así la transmisión de esfuerzos tectónicos en la litosfera ha sido analizada entre otros por Campbel (1978), Chinnery (1960), Johnston (1989), Johnston y Kanter (1990), Liu y Zoback (1977), Leroy y Machette (1996), Machette et al. (1991), Mackey et al. (1977), Stein (1999), Van de Plujim et al. (1997), Van Dusen y Doser (2000), y Zoback y Zoback (1981). A partir de ellos es conocido que: 1) la mayoría de los terremotos ocurren en los límites de placas; 2) no todos los sismos se producen en tales límites; 3) esos eventos, evidentemente, están en el interior de las placas; 3) la energía liberada por los primeros es muy superior a los del segundo grupo; 4) hay terremotos del interior de placa que poseen magnitudes similares a los mayores de la zona de entreplacas, pero con una frecuencia de repetición inferior. Tomando como base estos elementos se explican los terremotos de interior de placas a partir de: 1) la existencia de zonas de debilidad preneotectónicas; 2) la transmisión de los esfuerzos tectónicos desde las ZLP. Cotilla y Córdoba (2011b) explicaron así la ocurrencia del terremoto de San Cristóbal de 1880.

Los estudios sobre la sismotectónica de Cuba son diversos (Cotilla, 1993; Cotilla y Álvarez, 1999; Cotilla et al.,1991a; Orbera, 1983; Orbera et al., 1990). Cotilla (1998b) discute ampliamente sobre el tema. Y sobre la base de la ocurrencia de terremotos Cotilla y Franzke (1999) confirman la validez del mapa sismotectónico (MS) de Cotilla et al. (1991a). Este MS (Figura 4) permite la diferenciación de Cuba y su entorno inmediato. Es importante resaltar que para el caso de Cuba, nunca han sido determinadas rupturas en superficie por terremotos. También Cotilla et al. (1996) analizan y discuten todos los resultados publicados sobre neotectónica, sismotectónica y tectónica para Cuba, y producen un esquema similar al MS. Este 
material muestra un $93 \%$ de coincidencia. Cotilla et al. (2007) llegan a conclusiones similares con análisis de grupo. Además, se aseguró que la principal estructura sismogeneradora de Cuba es la FO. Esta falla (Cabo Cruz-Punta de Maisí) es un segmento del sistema de interacción de las placas de Norteamérica y Caribe. Ella ha sido dividida por Cotilla (1993) y Cotilla et al. (1998) en dos segmentos: 1) Occidental (Cabo Cruz-Baconao); 2) Oriental (Baconao-Punta de Maisí). Esa división se realizó sobre la base de un conjunto de 13 características neotectónicas y de datos sobre la sismicidad y los mecanismos focales. Esto se sustentó también a partir del análisis de fractales para las bahías de Santiago de Cuba (1.98) y Guantánamo (1.47), localizadas en los segmentos Occidental y Oriental, respectivamente (Cotilla y Córdoba, 2010a). Esa diferenciación se ajusta a la magnitud máxima determinada en ambas partes.

Cotilla et al. (1997a) indican, por primera vez, la diferenciación de la sismicidad y los mecanismos asociados en Cuba en tres regiones: Occidental, Oriental y Suroriental (Figura 4). La región Occidental corresponde a la típica SIP, en este caso de Norteamérica. La región Suroriental se corresponde con la sismicidad característica de entreplacas (Caribe y Norteamérica). Y la región de Cuba Oriental posee características e influencias de las dos regiones anteriores. Esto último se corresponde perfectamente con la propuesta de Scholtz et al. (1986).

Específicamente, los terremotos de 1880 (San Cristóbal= SC), 1914 (Gibara= G), 1939 (Remedios-Caibarién), 1974 (Esmeralda) y 1982 (Torriente-Jagüey Grande) (Figura 4) permiten realizar un análisis sismotectónico para entender la ocurrencia de terremotos en la zona de interior de placas de CO. Para esto se presentan las Tablas 5a, 5b, 7 y 8 que contienen algunos elementos de interés. En este sentido se comprueba que los terremotos de SC y G son los de mayor magnitud (6.2). Ellos se asocian a estructuras activas diferentes la FG y la FNC, respectivamente. La distancia entre ambos epicentros es de $\sim 825 \mathrm{~km}$. Desde el punto de vista neotectónico aunque están en la misma UNOC, el epicentro de SC está en una zona del interior de la isla y el de $\mathrm{G}$ en la zona marítima septentrional. El tiempo transcurrido entre ellos es de 34 años (Tabla 8).

La teoría actual de la Tectónica de Placas asegura que los eventos más fuertes se producen en las zonas de interacción y sus periodos de retorno o recurrencia son relativamente bajos. Los datos sobre los tres terremotos más fuertes de Cuba Suroriental (1766, 1852 y 1932) están en las Tablas 10a, 10b y la Figura 4. Estos eventos son de los mejor documentados (Cotilla, 2007). Se aprecia que las magnitudes son similares, y la $\mathrm{I}_{\text {máx }}=9$ (MSK) en uno de ellos en la ciudad de Santiago de Cuba. Los epicentros están aproximadamente a $15 \mathrm{~km}$ de esa ciudad. Los periodos de recurrencia entre ellos son de 76 y 80 años, respectivamente. El último de esos terremotos se produjo hace 81 años. Luego, desde el punto de vista estadístico un terremoto fuerte es probable ocurra en esa zona. Con todos estos elementos podemos suponer 
que: 1) el periodo de recurrencia de terremotos fuertes en San Cristóbal y Gibara es de $\sim 130$ años; 2) el área de ocurrencia de terremotos fuertes en Cuba Suroriental y $\mathrm{CO}$ es diferente, siendo mucho menor en la primera.

Aquí retomamos el comentario realizado en el epígrafe de Sismicidad sobre la FNC. Ella es una extensa estructura submarina $(>1,000 \mathrm{~km})$ que ha sido identificada como el límite Septentrional de megabloque Cuba, desde el Cabo de San Antonio a la Punta de Maisí (Figura 3). Esta falla tiene tres segmentos atendiendo a la dirección y configuración: 1) Oeste $=$ FNC1 (desde el Cabo de San Antonio hasta la Península de Hicacos); 2) Central= FNC2 (desde la Península de Hicacos hasta Cauto-Nipe); 3) Oriental= FNC3 (desde Cauto-Nipe hasta la Punta de Maisí). El segmento FNC1 es adyacente a la estructura oceánica del Golfo de México por el norte. El segmento FNC2 colinda con la parte más estrecha de la Plataforma de Bahamas. Mientras que el segmento FNC3 es aledaño a la Plataforma y al Océano Atlántico. Cada segmento está asociado con terremotos de los periodos histórico e instrumental (Occidental: serie de 1981; Central: 28 de febrero de 1914 (Ms=6.2), 15 de agosto de 1939 (Ms= 5.9), 25 de mayo de 1960; 18 de diciembre de 1986; Oriental: 5 de enero de 1990, 20 de marzo de 1992, 24 de septiembre de 1992, y 28 de diciembre de 1998). La FNC tiene la AS: 1) más fuerte en el segmento FNC2 (15 de agosto de 1939, Ms= 5.9 en Remedios-Caibarién; y 28 de febrero de 1914, $\mathrm{Ms}=6.2$ en Gibara); 2) menor en el segmento FNC1. En este último segmento es donde se localiza el cambio de dirección de la $\mathrm{FNC}$, en esa área se han definido nudos sismoactivos (Figura 4), además, la FNC entronca con las FO y del norte de La Española. Allí configura el nudo sismoactivo N3 (Cotilla, 1993; Cotilla et al., 1998) con múltiples terremotos (Figura 6c).

Con independencia de que los datos sobre tsunamis en Cuba, en cuanto a localización e importancia, han tenido distorsiones significativas (Cotilla, 2011; Cotilla y Córdoba, 2011a); ellos se asocian, únicamente, a la costa norte, por ejemplo el de 1939 (Cotilla, 2011). Esto conlleva a asumir que los sistemas de fallas son de tipo normal e inverso, principalmente. Indicamos que el tsunami de 1946 en República Dominicana también se produjo en la zona norte, Península de Samaná.

Se ha determinado que la mayor parte de las fallas de la parte insular de Cuba son espacialmente análogas, en cuanto a dirección, a la FNC. Así en Pinar del Río la FG tiene dirección NE y es paralela al segmento FNC1. Las FLV y FC, que pertenecen también a la misma UN que la FG, son paralelas al segmento FNC2. Todas ellas son sísmicamente activas y responden a un mismo patrón de esfuerzos, sin embargo, en la UNOR no hay datos sobre la existencia de alguna estructura sísmicamente activa paralela a la FNC.

Por último, se presenta la Tabla 12 con un resumen de las características determinadas para las zonas de entreplacas y de interior de placa en Cuba se sostiene que Cuba es el único territorio del Caribe Septentrional con estos tipos de sismicidad. 
Tabla 12

Características de las zonas sísmicas cubanas

\begin{tabular}{lcc}
\hline & \multicolumn{2}{c}{ Sismicidad } \\
\multicolumn{1}{c}{ Características } & Entreplacas & Interior de placa \\
\hline Magnitud máxima / Profundidad máxima $(\mathrm{km})$ & $6.8 / 60$ & $6.2 / 20$ \\
Distancia máxima entre eventos fuertes $(\mathrm{km})$ & 100 & 825 \\
Periodo de repetición (años) & $\sim 80$ & $>100$ \\
Área máxima de afectación $\left(\mathrm{km}^{2}\right)$ & 110,000 & 40,000 \\
Distancia máxima de perceptibilidad $(\mathrm{km})$ & $\sim 800$ & $\sim 300$ \\
Epicentro & Mar & Tierra y mar \\
Isosistas & Medias & Completas y medias \\
Intensidad máxima (MSK) & 9 & 8 \\
Estimado de la ruptura máxima $(\mathrm{km})$ & 85 & 58 \\
Terremotos producidos $(\%)$ & $\sim 75$ & $<25$ \\
Rangos de magnitud / cantidad de terremotos & $6.8-6.0 / 6 ; 5.9-5.0$ & $6.2-6.0 / 2 ; 5.9-5.0 /$ \\
& $/ 18$ & 3 \\
Cantidad de muertos & $>100$ & $\sim 20$ \\
\hline
\end{tabular}

\section{Conclusiones}

En Cuba hay tres tipos de sismicidad: 1) entreplacas por la interacción CaribeNorteamérica (la más fuerte, frecuente e importante). Ella se corresponde con la Unidad Sismotectónica de Cuba Suroriental; 2) interior de la placa Norteamérica (con la mayor área) que se corresponde con la Unidad Sismotectónica Occidental; 3) intermedia en la Unidad Sismotectónica de Cuba Oriental, y que espacialmente limita con las dos zonas anteriores. Ella tiene características predominantes del tipo interior de placas.

En la Unidad Neotectónica Occidental hay 900 terremotos perceptibles (14922013). El valor de la $M_{\text {máx }}$ estimada es 6.2, años 1880 y 1914. Los terremotos más fuertes indican un periodo de recurrencia superior a 100 años. Estos eventos han producido algunos muertos y heridos, pero mucho menos que los de la región suroriental ( $\sim 100)$. La actividad sísmica se justifica por la influencia dinámica del límite de placas Caribe-Norteamérica en las zonas de fallas y sus intersecciones.

\section{Agradecimientos}

A los profesores Diego Córdoba Barba y Miguel Herraiz Sarachaga por la revisión del manuscrito original. El trabajo se realizó en el Departamento de Física de la Tierra, Astronomía y Astrofísica 1. Amador García Sarduy preparó todas las figuras. El trabajo ha sido parcialmente financiado por cuatro proyectos: TSUJAL (CGL2011-29474-C02-01), CARIBENORTE (CTM2006-13666-C02-02), TOPOIBERIA (CSD2006/0041), y GR35/10-A/910549. 


\section{Referencias}

Álvarez L.; Cotilla M. y Chuy T., 1990. Informe final del tema 430.03: Sismicidad de Cuba. Archivo del Departamento de Sismología, Instituto de Geofísica y Astronomía, Academia de Ciencias de Cuba.

Álvarez L., Rubio M., Chuy T. y Cotilla M., 1985. Informe final del tema de investigación 31001: Estudio de la sismicidad de la región del Caribe y estimación preliminar de la peligrosidad sísmica en Cuba. Archivo del Departamento de Sismología, Instituto de Geofísica y Astronomía, Academia de Ciencias de Cuba.

Álvarez L., Chuy T., García J., Moreno B., Álvarez H., Blanco M., Expósito O., González O. y Fernández A.I., 1999. An earthquake catalogue of Cuba and neighbouring areas. The Abdus Salam International Centre for Theoretical Physics, Miramare-Trieste, Internal Report IC/IR/99/1, 60 pp. (en inglés).

Arsovsky M. y Hadzievsky D., 1970. Correlation between neotectonics and the seismicity of Macedonia. Tectonophysics, 9, 129-142 (en inglés).

Assinovskaya B.A. y Soloviev S.L., 1994. Definition and description of the zones of potential earthquake sources in the Barents Sea. Izvestiya Physics of the Solid Earth., 29(8), 664-675 (en inglés).

Backmanov D.M. y Rasskazov A.A., 2000. Recent faults in the junction área between the southern and central Urals. Geotectonics, 4, 25-31 (en inglés).

Balassanian S.Y., Arakelian A.R., Nazaretian S.N., Avanessian A.S., Martirossian A.H., Igoummov V.A., Melkounian M.G., Manoukian A.V. y Tovnassian A.K., 1995. Retrospective analysis of the Spitak earthquake. Annali di Geofisica, 38(3-4), 345-373 (en inglés).

Campbell D.L., 1978. Investigation of the stress concentration mechanism for intraplate earthquakes. Geophys. Res. Lett., 5, 477-479 (en inglés).

Chinnery M.A., 1960. The stress changes that accompany strike-slip faulting. Bull. Seism. Soc. Am., 53, 921-932 (en inglés).

Chuy T. , 1989. Isosistas de terremotos de Cuba. Nuevo Atlas Nacional de Cuba. Sección Características Geofísicas, II.3.2. Madrid.

,2003. Macrosísmica de Cuba: Su aplicación en los estudios de Peligrosidad Sísmica. Minería y Geología, 1-2, 43-50.

Chuy T., González B. y Álvarez L., 1980. Sobre la peligrosidad sísmica en Cuba. Revista Investigaciones Sismológicas en Cuba, 4, 37-52.

Chuy T., González B. y Escalona S., 1980. Información macrosísmica de las provincias de Villa Clara, Cienfuegos y Sancti Spíritus. Revista Investigaciones Sismológicas en Cuba, 1, 33-57. 
Chuy T., González B. y Polo B., 1988. Algunos criterios sobre la peligrosidad sísmica de la región occidental de Cuba. Comunicaciones Científicas sobre Geofisica y Astronomía, 4, 19 p.

Chuy T., González B. y Vorobiova E., 1984. Sismicidad del territorio de las provincias de Camagüey y Ciego de Ávila, Cuba. Revista Investigaciones Sismológicas en Cuba, 5, 61-94.

Chuy T., Vorobiova E., González B., Álvarez L., Pérez E., Serrano M., Cotilla M. y Portuondo O., 1983b. El sismo del 16 de diciembre de 1982. Torriente-Jagüey Grande. Revista Investigaciones Sismológicas en Cuba, 3, 43.

Cotilla M.O., 1999. El controvertido alineamiento Habana-Cienfuegos, Cuba. Estudios Geológicos, 55(1-2), 67-88.

- 2003. The Santiago de Cuba earthquake of 11 June 1766: Some new insights. Geofísica Internacional, 42(4), 589-602 (en inglés).

— 2007 . Un recorrido por la Sismología de Cuba. Editorial Complutense, Madrid.

— 2011. ¿Tsunamis en Cuba? Revista Física de la Tierra, 23, 173-197.

Cotilla M., 1993. Una caracterización sismotectónica de Cuba. PhD Thesis, Instituto de Geofísica y Astronomía, Academia de Ciencias de Cuba, 200 pp.

- 1998. Una revisión de los estudios sismotectónicos en Cuba. Revista Estudios Geológicos, 54(3-4), 129-145.

— 1998a. Sismicidad y sismotectónica de Cuba. Revista Física de la Tierra, $10,53-86$.

$\longrightarrow, 1998$ c. Terremotos de Cuba. GEOS, 18(3), 180-188.

- , 1998c. An overview on the seismicity of Cuba. Journal of Seismology, 2, 323-335 (en inglés).

Cotilla M.O. y Álvarez J.L., 1999. Mapa de zonas sismogeneradoras de Cuba. Geología Colombiana, 23, 97-106.

—, 2001. Regularidades sismogenéticas de la unidad neotectónica Occidental de Cuba. Revista Geológica de Chile, 28(1), 3-24.

Cotilla M. y Córdoba D., 2003. Caracterización morfotectónica de Galicia, España. Revista Geofísica, 58, 5-56.

- 2004. Morphotectonic of Iberian Penynsula. Pure appl. geophys., 161(4), 755-815 (en inglés).

- 2008. Notes on three earthquakes in Santiago de Cuba (14.10.1800, 18.09.1826, 07.07.1842). Russian Geology and Geophysics, 51, 243-251.

- 2010a. Study of the Cuban fractures. Geotectonics, 44(2), 176-202 (en inglés).

- , 2010b. The Bayamo earthquake (Cuba) of the 18 October 1551. International Journal of Geosciences, 1, 1-13 (en inglés). 
, 2010c. The August 20, 1852 earthquake in Santiago de Cuba. Russian Geology and Geophysics, 51, 1,227-1,246 (en inglés).

, 2011a. Comments about tsunami occurrences in the northern Caribbean. Tsunamis (en inglés).

- 2011b. Study of the earthquake of the January 23, 1880, in San Cristóbal, Cuba and the Guane fault. Izvestiya Physics of the Solid Earth, 47(6), 496-518 (en inglés).

Cotilla M., Bankwitz P., Franzke H.J., Álvarez L., González E., Díaz J.L., Grünthal G., Pilarski J. y Arteaga F., 1991a. Mapa sismotectónico de Cuba, escala 1:1,000,000. Comunicaciones Científicas sobre Geofisica y Astronomía, 23, 35 pp.

Cotilla M.O., González E.C., Franzke H.J., Díaz J.L., Arteaga F. y Álvarez L., 1991b. Mapa neotectónico de Cuba, escala 1:1,000,000. Comunicaciones Cientificas sobre Geofisica y Astronomía, 22, $37 \mathrm{pp}$.

Cotilla M., Millán G., Álvarez L., González D., Pacheco M. y Arteaga F., 1996. Esquema neotectogénico de Cuba. Informe científico-técnico del Departamento de Geofísica del Interior. En Archivo del Instituto de Geofísica y Astronomía, Academia de Ciencias de Cuba, 100 pp.

Cotilla M., Álvarez L. y Rubio M., 1997A. Sismicidad de tipo intermedio en Cuba. Revista Geología Colombiana, 22, 35-40.

Cotilla M., Rubio M., Álvarez L. y Grünthal G., 1997b. Potenciales sísmicos del sector Centro-Occidental del arco de las Antillas Mayores. Revista Geofisica, 46, 129-150.

Cotilla M. y Franzke H.J., 1999. Validación del mapa sismotectónico de Cuba. Boletín Geológico y Minero, 110(5), 573-580.

Cotilla M.O. y Udías A., 1999. Ge4odinámica del límite Caribe-Norteamérica. Rev. Soc. Geol. de España, 12(2), 175-186.

Cotilla M.O., Franzke H.J. y Córdoba D., 2007. Seismicity and seismoactive faults of Cuba. Russian Geology and Geophysics, 48, 505-522 (en inglés).

Cotilla M., Bankwitz P., Álvarez L., Franzke H.J., Rubio M.F. y Pilarski J., 1998. Cinemática neotectónica de Cuba. Rev. Soc. Geol. de España, 11(1-2), 33-42.

Erickson A.J., Helsley C.E. y Simmons G., 1972. Heat flow and continuous seismic profiles in the Cayman trough and Yucatan basin. Geol. Soc. Am. Bull., 83, 1,241-1,260 (en inglés).

Evison F.F., 1963. Earthquakes and faults. Bull. Seism. Soc. Am., 53, 873-891 (en inglés).

Frohlich C., 1982. Seismicity of the Central Gulf of Mexico. Geology, 10, 103-106 (en inglés).

Gianella V.P., 1957. Earthquakes and faulting, Fort Sage Mountains, California, December 1950. Seism. Soc. Am. Bull., 47, 173-177 (en inglés). 
González B. y Chuy T., 1983. Actividad sísmica de la Provincia Pinar del Río. Revista Investigaciones Sismológicas en Cuba, 4, 53-68.

González B.E., Álvarez J., Serrano M., García J., Rodríguez V., Pérez L. y Fernández E., 1995. Informe científico-técnico del 9 de marzo de 1995: Ganuza, Municipio San José de las Lajas. En Archivo del Centro Nacional de Investigaciones Sismológicas, Filial Occidental. Ministerio de Ciencia, Tecnología y Medio Ambiente de Cuba, 13 pp.

González E.C., Cotilla M.O., Cañete C.C., Díaz J.L., Carral R. y Arteaga F., 2003. Estudio morfoestructural de Cuba. Geogr. Fis. Dinam. Quat., 26(1), 49-70.

Griggs D. y Handin J., 1960. Observations on fractures and hypothesis of earthquakes. Geol. Soc. Am. Mem., 79, 347-364 (en inglés).

Guelfand I.M., Guberman S.A., Keylis-Borok V.I., Knopoff L., Press F.S., Rantsman E.Y., Rotvain I.M. y Skii A.M., 1976. Condiciones para el surgimiento de terremotos fuertes (California y otras regiones). Vichislitielnaya Seismologiya, 9, 3-91 (en ruso).

Gutiérrez Lanza M., 1914. Conferencias de sismología pronunciadas en la Real Academia de Ciencias de La Habana. Editorial Imprenta y Librería de Lloredo y Cña., La Habana, 178 pp.

Gutiérrez Lanza M., 1910. Puntos de vista sobre los terremotos. Trabajo enviado al IV Congreso Científico, vol. V, 5 p., Santiago de Chile.

Gvshiani A.D., Zelevinsky A.V., Keilis-Borok V.I. y Kosobokov V.G., 1978. Estudio de las áreas de ocurrencia de terremotos fuertes en el Cinturón del Océano Pacífico con ayuda de algoritmos de reconocimiento. Fizika Zemli, 9, 31-42 (en ruso).

, 1980. Reconocimiento de las áreas donde pueden ocurrir los terremotos más fuertes $(\mathrm{Ms} \geq 8.2)$. Métodos y algoritmos para la interpretación de datos sismológicos. Vichislitielnaya Seismologiya, 13, 30-43 (en ruso).

Gvshiani A.D., Gorshkov A., Kosobokov V., Cisternas A., Philip H. y Weber C., 1987. Identification of seismically dangerous zones in the Pyreness. Annales Geophysicae, 87, 681-690 (en inglés).

Hadmut K.W., Sieber L. y Pacheco J., 1989. Cross-fault triggering in the November 1987. Superstition Hills earthquake sequence, southern California. Geophys. Res. Lett., 16, 199-203 (en inglés).

Johnston A.C., 1989. The seismicity of stable continental interiors. En Earthquakes at North Atlantic Passive Margins: neotectonics and postglacial resound, 229327 (Editores: S. Gregersen y P.W. Bashau) (en inglés).

Johnston A.C. y Kanter L.R., 1990. Earthquakes in stable continental crust. Scientific American, 10, 42-49 (en inglés).

Leonov Yu G., 1995. Esfuerzos en la litosfera y geodinámica de interior de placa. Geotectonika, 6, 3-22 (en ruso). 
Leroy S.D., 1998. Treating the Gulf of Mexico as a continental margin petroleum province. The Leading Edge, 209-212 (en inglés).

Leroy S. y Mauffret A., 1996. Intraplate deformation in the Caribbean region. $J$. Geodynamics, 21(1), 113-122 (en inglés).

Liu L. y Zoback M.D., 1977. Lithospheric strenghts and intraplate seismicity in the New Madrid seismic zone. Tectonics, 16, 585-595 (en inglés).

Machette M., Personius S., Nelson A., Schwartz D. y Lund D., 1991. The Wasatch fault zone, Utah: segmentation and history of the Holocene earthquakes. $J$. Struct. Geol., 13(2), 137-149 (en inglés).

Mackey K.G., Fiyita K., Gunbina L.V., et al., 1977. Seismicity of the Bering Strait region: Evidence for Bering block. Geology, 25, 979-982 (en inglés).

Makarov G.V. y Schukin Y.K., 1976. Valoración de la actividad de las fallas ocultas. Geotektonika, 1, 96-109 (en ruso).

Mann P. y Burke K., 1984. Neotectonics of the Caribbean. Rev. Geophys. Space Phys., 22, 309-362, (en inglés).

Meyer B., Armijo R. y Dimitrov D., 2002. Active faulting in SW Bulgaria: Possible surface rupture of the 1904 Struma earthquake. Geophys. J. Int., 148, 246-255 (en inglés).

Mogi K., 1967. Earthquakes and fractures. Tectonophysics, 5, 35-55 (en inglés).

Montoulieu E.L., 1932. El megasismo de Santiago de Cuba del 3 de febrero de 1932 bajo la luz de la Sismología Moderna, La Habana, 100 pp.

—, 1947. Aspecto geográfico del macrosismo sentido el 15 de agosto de 1939 en la región Central de Cuba. Revista de la Sociedad Cubana de Ingenieros, 20(1-2), La Habana.

- 1968. Notas y apuntes acerca de terremotos ocurridos en Cuba. Inédito. Fondos del Instituto de Geofísica y Astronomía, Academia de Ciencias de Cuba.

Morales y Pedroso L., 1931. Los terremotos de Cuba. Revista de la Sociedad Cubana de Ingenieros, 5, 264-308.

Moreno Toirán B., 2002. The new Cuban seismograpgh network. Seism. Res. Lett., 33(4), 504-517 (en inglés).

Moreno B., Grandison M. y Atakan K., 2002. Crustal velocity model along the southern Cuban margin: Implications for the tectonic regime at an active plate boundary. Geophys. J. Int., 151, 632-645 (en inglés).

Morgan W.J., 1968. Rises, trenches, great faults and crustal blocks. J. Geophys. Res., 73, 1,551-1,982 (en inglés).

Ofoegbu G.I. y Ferrill D.A., 1998. Mechanical analysis of listric normal faulting with emphasis on seismicity assessment. Tectonophysics, 284, 65-77 (en inglés).

Orbera L., 1983. Estudio sismotectónico de la región Occidental. En: Archivos de la Empresa Integral de Proyectos de la Industria Básica, Ministerio de la Industria Básica de Cuba, 62 pp. 
Orbera L., González B., Chuy T. y Oro J., 1990. Investigaciones sísmicas en la región de emplazamiento del Centro de Investigaciones Nucleares. Vol. 1. Secretaría Ejecutiva para Asuntos Nucleares de Cuba, Academia de Ciencias de Cuba. 344 pp.

Orowan E., 1960. Mechanism of seismic faulting. Geol. Soc. Am. Mem., 79, 333345 (en inglés).

Pavlides S.B., Zouros N.C., Zhongüng F., Shaoping C. y Chatzipetros A.A., 1999. Geometry kinematics and morphotectonics of the Yanquing-Huailai active faults (northern China). Tectonophysics, 308, 99-118 (en inglés).

Poey A., 1855a. Tableau chronologique des tremblements de terre ressentis a I'ile de Cuba de 1551 á 1855. Annales des Voyages, 6 a serie, 11, 301 p. Malte-Brun, Paris, (en francés).

- $1855 \mathrm{~b}$. Supplément au tableau chronologique des tremblements de terre ressentis a I'ile de Cuba de 1530 á 1855. Annales des Voyages, 4, 286 p. MalteBrun, Paris, (en francés).

- 1857. Catalogue chronologique des tremblements de terre ressentis dan les Indes Occidenteles de 1530 á 1857. Accompagné d'une Revue Bibliographique Contenant Tous les Travaux Relatife aux Tremblements de Terre des Antilles. Annuarie de la Societé Météorologique de France, 5, 75-227, Paris (en francés).

Potilla J.A. y Anderson K.B., 2004. Fault interaction at the junction of transverse Range and Eastern California shear zone: A case study of intersecting faults. Tectonophysics, 379, 43-60 (en inglés).

Prol J., Ariaza G. y Otero R., 1993. Sobre la confección de los mapas de profundidad del basamento y espesor de la corteza terrestre en el territorio cubano. Informe Científico-Técnico de la Empresa Nacional de Geofísica, Ministerio de la Industria Básica de Cuba, 36 pp.

Randall M.J., 1964. On the mechanism of earthquakes. Bull. Seism. Soc. Am., 54, 1,283-1,289 (en inglés).

Rosencratz E., 1990. Structure and tectonic of the Yucatan basin, Caribbean Sea, as determine from seismic reflection studies. Tectonics, 9(5), 1,037-1,059 (en inglés).

Rosencratz E., Ross M.I. y Sclater J.G., 1988. Age and spreading history of the Cayman trough as determined from depth, heat flow and magnetic anomalies. $J$. Geophys. Res., 93, 2,141-2,157 (en inglés).

Rowan L.C. y Wetlaufer P.H., 1981. Relation between regional lineament systems and structural zones in Nevada. Am. Assoc. Pet. Geol. Bull., 65(8), 1,414-1,432 (en inglés).

Rubio M., 1982. Ocurrencia de tsunamis en el Caribe. Revista Investigaciones Sismológicas en Cuba, 2, 170-180. 
Russo R.M. y Vilasenor A., 1995. The 1946 Hispaniola earthquake and the tectonics of the North America-Caribbean plate boundary zone, northeastern Hispaniola. J. Geophys. Res., 100, 6,265-6,280 (en inglés).

Salteraín y Legarra P., 1884. Ligera reseña de los temblores de tierra ocurridos en la Isla de Cuba. Anales de la Academia de Ciencias Médicas, Físicas y Naturales de La Habana, 21, 203-218.

Scholtz C.H., Aviles C.A. y Wesnousky S.G., 1986. Scaling differences between large interplate and intraplate earthquakes. Bull. Seism. Soc. Am., 76(1), 65-70 (en inglés).

Scwartz D.P. y Coppersmith K.J., 1986. New trends in analysis using geologic data. Chapter 14. Active Tectonics. Studies in Geophysics, National Academic Press, 215-230 pp. (en inglés).

Sherbakova B.E., Bovenko V.G., Luzenko T.N., Miroschinichenko I.P., Pozniakova G.V. y Zharinova N.I., 1977. Informe sobre los resultados de observaciones con los aparatos "Tierra" en el territorio de Cuba Oriental realizados en 19741975. Archivo del Instituto de Física de la Tierra, vol. 1, Moscú (en ruso).

Sibson R.H., 1985. A note on fault reactivation. J. Struct. Geol., 7, 751-754 (en inglés).

Sim L.A., 1991. Estudios de los esfuerzos tectónicos a partir de indicadores geológicos (métodos, resultados, y recomendaciones). Izv. Vysch. Uchebn. Zaved, Geol. Razved, 10, 3-23 (en ruso).

Smith R.B. y Sbar M.L., 1974. Contemporary tectonics and seismicity of the western United States with emphasis in the intramountain seismic belt. Geol. Soc. Am. Bull., 85, 1,205-1,218 (en inglés).

Smoot N.Ch., 1997. Aligned buoyant highs, across-trench, clustered volcanoes, and deep earthquakes are not aligned with plate-tectonic theory. Geomorphology, 18, 199-222 (en inglés).

Stein R.S., 1999. The role of stress transfer in earthquake occurrence. Nature, 402, 605-609 (en inglés).

Sykes L.R., 1978. Intraplate seismicity reactivation of pre-existing zones of weakness, alkaline magmatism and tectonic postdating continental fragmentation. Reviews of Geophysics and Space Physics, 16(4), 621-688 (en inglés).

Tchalenko J.S., 1970. Similarities between shear zones of different magnitudes. Geol. Soc. Am. Bull., 81, 1,625-1,640 (en inglés).

Teisseyre R., 1990. Crack formation and energy release caused by the concentration of dislocations along fault planes. Tectonophysics, 9, 547-557 (en inglés).

USGS, 2006. Earthquakes list of 2006.

Van der Plujim B.A., Craddock J.P., Graham B.R. y Harris J.H., 1997. Paleostress in cratonic North-America: Implications for deformation of continental interiors. Science, 277, 794-796 (en inglés). 
Van Dusen S.R. y Doser D.I., 2000. Faulting process of historic (1917-1962) M $\geq$ 6.0 earthquake along the North-Central Caribbean margin. Pure appl. geophys., $157,719-736$ (en inglés).

Viñes B. y Salteraín P., 1880. Excursión a Vuelta Abajo de Viñes y Salteraín en ocasión de los fuertes temblores de tierra ocurridos en la noche del 22 al 23 de enero de 1880. Ediciones La Voz de Cuba, La Habana, 68 pp.

Wallace R.E., 1984. Patterns and timing of the Late Quaternary faulting in the Great Basin Province and relation the some regional tectonic features. $\mathrm{J}$. Geophys. Res., 89, 5,763-5,769 (en inglés).

Wells D.L. y Coppersmith K.J., 1994. New empirical relationships among magnitude, rupture length, rupture wide, rupture area, and surface displacement. Bull. Seism. Soc. Am., 84, 974-1,002 (en inglés).

Wernicke B., 1995. Low-angle normal faults and seismicity: A review. J. Geophys. Res., 100, 20,159-20,174 (en inglés).

Wdowinski, 1998. A theory of intraplate tectonics. J. Geophys. Res., 103, 5,0375,059 (en inglés).

Wyss M., 1979. Estimating maximum expectable magnitude of earthquakes from fault dimensions. Geology, 7, 336-340 (en inglés).

Zhidkov M.P., Rotvain I.M. y Sadowskii A.M., 1975. Pronóstico del lugar de posible ocurrencia de terremotos fuertes. IV. Intersecciones múltiples de lineamientos de la Meseta Armeniana, los Balkanes y la Cuenca del Mar Egeo. Vichislitelnaya Seismologiya, 8, 53-70 (en ruso). 